\title{
Development of a Meso-Scale Material Model for Ballistic Fabric and Its Use in Flexible-Armor Protection Systems
}

\author{
M. Grujicic, W.C. Bell, G. Arakere, T. He, X. Xie, and B.A. Cheeseman
}

(Submitted February 14, 2009)

\begin{abstract}
A meso-scale ballistic material model for a prototypical plain-woven single-ply flexible armor is developed and implemented in a material user subroutine for the use in commercial explicit finite element programs. The main intent of the model is to attain computational efficiency when calculating the mechanical response of the multi-ply fabric-based flexible-armor material during its impact with various projectiles without significantly sacrificing the key physical aspects of the fabric microstructure, architecture, and behavior. To validate the new model, a comparative finite element method analysis is carried out in which: (a) the plainwoven single-ply fabric is modeled using conventional shell elements and weaving is done in an explicit manner by snaking the yarns through the fabric and (b) the fabric is treated as a planar continuum surface composed of conventional shell elements to which the new meso-scale unit-cell based material model is assigned. The results obtained show that the material model provides a reasonably good description for the fabric deformation and fracture behavior under different combinations of fixed and free boundary conditions. Finally, the model is used in an investigation of the ability of a multi-ply soft-body armor vest to protect the wearer from impact by a 9-mm round nose projectile. The effects of inter-ply friction, projectile/ yarn friction, and the far-field boundary conditions are revealed and the results explained using simple wave mechanics principles, high-deformation rate material behavior, and the role of various energyabsorbing mechanisms in the fabric-based armor systems.
\end{abstract}

Keywords ballistic performance, flexible armor, high-performance fibers, meso-scale unit-cell material model

\section{Introduction}

In order to respond to the new enemy threats and warfare tactics, military systems, in particular those supporting the U.S. ground forces, are being continuously transformed to become faster, more agile, and more mobile so that they can be quickly transported to operations located throughout the world. Consequently, an increased emphasis is being placed on the development of improved lightweight body-armor and lightweight vehicle-armor systems as well on the development of new high-performance armor materials. High-performance fiber-based materials have been exploited for both body-armor (e.g., as soft, flexible fiber mats for personal-armor vests) and for the vehicle-armor systems (e.g., as reinforcements in rigid polymer matrix composites, PMCs, for lightweight vehiclearmor systems).

M. Grujicic, W.C. Bell, G. Arakere, T. He, and X. Xie, International Center for Automotive Research CU-ICAR, Department of Mechanical Engineering, Clemson University, 241 Engineering Innovation Building, Clemson, SC 29634-0921; and B.A. Cheeseman, Army Research Laboratory-Survivability Materials Branch, Proving Ground, Aberdeen, MD 21005-5069. Contact e-mail: mica.grujicic@ces.clemson. edu.
Flexible lightweight materials have been used, throughout history, in body-armor systems to provide protection against specified threats, at reduced weight and without compromising person's mobility. Early materials used included leather, silk, metal chain mail, and metal plates. Replacement of metal with a nylon (poly-amide) fabric and an E-glass fiber/ethyl cellulose composite in body-armor systems can be linked to the Korean War (Ref 1). Although, primarily due to their low cost, nylon and E-glass fibers are still being used today, high-performance polymeric fibers (typically used in the form of woven fabrics) are now the standard in most fiber-reinforced body-armor applications. To increase the ballistic performance of the bodyarmor vests relative to up to 0.30 caliber threats, ceramic insert strike-plates are commonly used (Ref 2).

The defining characteristics of high-performance polymeric fibers used today are their superior strength, stiffness and ballistic performance. Among these high-performance fibers the most notable are: (a) poly-aramids (e.g., Kevlar ${ }^{\circledR}$, Twaron ${ }^{\circledR}$, Technora ${ }^{\circledR}$ ); (b) highly oriented poly-ethylene (e.g., Spectra ${ }^{\circledR}$, Dyneema ${ }^{\circledR}$ ); (c) poly-benzobis-oxazole, PBO (e.g., Zylon ${ }^{\circledR}$ ), and (d) poly-pyridobisimi-dazole, PIPD (e.g., M5 ${ }^{\circledR}$ ). When tested in tension, all these materials differ significantly from the nylon fibers, having very high absolute stiffness, extremely high density-normalized strength, and quite low $(<4 \%)$ strainsto-failure. These fibers essentially behave, in tension, as rateindependent linear elastic materials. When tested in transverse compression, however, these fibers are similar to nylon and can undergo large plastic deformation without a significant loss in their tensile load-carrying capacity. This behavior is quite different from that found in carbon or glass fibers, which tend to shatter under transverse compression loading conditions. 
In today's practice, fabrics based on high-performance fibers are extensively employed in variety of ballistic and impact protection applications. Despite the fact that over the past two decades, there has been a great deal of work done on understanding the ballistic behavior of these fabrics using various analytical and numerical techniques, the design of fabric armor systems remains largely based on the employment of extensive experimental test programs, empiricism and old practices. While such experimental programs are critical for ensuring the utility and effectiveness of the armor systems, they are generally expensive, time-consuming and involve destructive testing. Consequently, there is a continuing effort to reduce the extent of these experimental test programs by complementing them with the corresponding computation-based engineering analyses and simulations.

A review of the literature carried out as part of the present work, revealed the following main classes of computationalengineering analyses used to model ballistic performance of flexible armor: (a) Finite element analyses based on the use of pin-jointed orthogonal bars to represent flexible fabric yarns. The most notable studies falling into this category of analyses are those performed by Roylance and Wang (Ref 3), Shim et al. (Ref 4), Lim et al. (Ref 5), Shahkarami et al. (Ref 6), Johnson et al. (Ref 7), and Billon and Robinson (Ref 8). While the pinjointed orthogonal-bars based finite element analyses have proven to be very efficient in approximating the dynamic behavior of woven fabrics, the discrete nature of the yarn models was associated with inherent oversimplifications that significantly limited the predictive capability of the analyses. In particular, important contributions associated with the weave architecture, surface-finish and friction governed yarn-to-yarn and layer-to-layer contacts (in multi-layer fabrics) could not be accounted for; (b) more-detailed full-blown three-dimensional (3D) continuum finite element analyses such as the ones carried out by Shockey et al. (Ref 9), Duan et al. (Ref 10-13), Zhang et al. (Ref 14), etc. have also been investigated. While these analyses have proven to be powerful tools for capturing and elucidating the detailed dynamic response of single-layer fabrics, they are computationally very demanding when applied to practical armor systems which typically contain 30-50 fabric layers/plies; (c) unit-cell based approaches have been used extensively in order to derive the equivalent (smeared) continuum-level (membrane/shell) material models of textile composites from the knowledge of the meso-scale fiber and yarn properties, fabric architecture and inter-yarn and inter-ply frictional characteristics. Among the most notable studies based on these analyses are those carried out by Kawabata et al. (Ref 15-17) who introduced simple analytical models to capture the uniaxial, biaxial, and shear behavior of fabrics. Furthermore, Ivanov and Tabiei (Ref 18) proposed a micromechanical material model for a woven fabric (in which a visco-elastic constitutive model was used to represent the mechanical behavior of the yarns) for the use in nonlinear finite element impact simulations. In deriving the material model, Ivanov and Tabiei (Ref 18) considered the motion of the yarn-crossover point and developed a procedure for determining the equilibrium position of this point under the applied unit-cell strains. Recently, King et al. (Ref 19) proposed a new approach for deriving the continuum-level material model for fabrics based on the properties of the yarns and the weave architecture which involves the use of an energy minimization technique to establish the relationship between the configurations of the fabric structure to the microscopic deformation of fabric components. Similar unit-cell based continuum-level membrane/shell material models have been developed by Boisse et al. (Ref 20) and Peng and Cao (Ref 21). Also, Shahkarami and Vaziri (Ref 22) proposed a similar but simpler model to that introduced by King et al. (Ref 19) and provided a detailed account of its incorporation into a material-model subroutine which can be readily coupled with commercial dynamicexplicit finite element codes; and (d) the use of higher order membrane/shell finite element analyses to represent the dynamic response of fabric under ballistic loading conditions and overcome the aforementioned computational cost associated with the use of full 3D finite element analyses of the yarn/ fabric structure. Among the studies falling into this category, the most notable is the one carried out by Scott and Yen (Ref 23). While the use of higher order membrane elements was found to be indeed advantageous computationally, it was never fully validated by comparing its results against either those obtained experimentally or those obtained using full 3D finite element analyses.

As mentioned earlier, while major efforts have been made in recent years to develop sophisticated numerical models capable of elucidating the ballistic performance of fabric armors, most of these models either lack computational efficiency or fail to capture many physical aspects of the yarn and fabric architecture and/or contact dynamic phenomena. Hence, the main objective of the present work is to develop an efficient shell-based meso-scale mechanics unit-cell based model that captures the essential dynamic/ballistic behavior of plain woven fabric under impact-loading conditions. The term "meso-scale" is used to denote yarn-level millimeter length scale details of the fabric microstructure/architecture. In other words, finer-scale molecular-level and fiber-level material details are not considered explicitly and instead only their lumped contributions are taken into account. The "unit-cell" term is used to denote the basic structural unit in a woven single-ply fabric so that a fabric patch can be considered as an in-plane assembly of such units. The material model developed in the present work is essentially an extension of the model recently proposed by Shahkarami and Vaziri (Ref 22) and includes all the cases of in-plane unit-cell deformation modes and, for each of these modes, all the contact/no-contact cases of the crossing yarns. In addition, 3D single unit cell finite element analyses of different in-plane and transverse loading scenarios were carried out to assess the yarn/yarn contact force versus over-closure relationship, as well as the in-plane and out-of-plane shear moduli. The contact force versus over-closure relationship and the in-plane and out-ofplane shear unit-cell moduli are then used as input in the meso-scale unit-cell based material model.

The organization of the paper is as follows: Details regarding the computational procedures employed to develop a new meso-scale unit-cell based material model for a prototypical plain-woven single-ply fabric and the implementation of this model into a material user subroutine suitable for use in commercial finite element programs are presented in Section 2. The formulation of a simple projectile-armor impact problem used to validate the new material model is described in Section 3. Main results obtained in the current work are presented and discussed in Section 4. Application of the new meso-scale unit-cell based material model in the analysis of personnel protection is presented in Section 5. The main summary points and conclusions resulting from the present work are listed in Section 6. 


\section{Model Development and Computational Procedure}

Details regarding the development of a simple meso-scale unit-cell based material model for a prototypical plain-woven

(a)

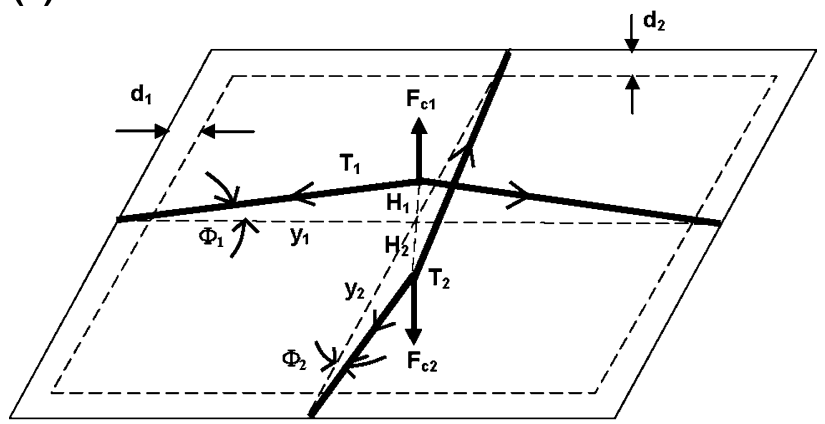

(b)

Fig. 1 Geometrical representation of a single unit cell for a plainwoven single-ply fabric: (a) before and (b) after application of a normal biaxial in-plane deformation single-ply fabric and its implementation into a material-user subroutine suitable for use in commercial finite element packages are presented in this section. A simple schematic of the unit cell which is used to represent the plain-woven singleply fabric structure/architecture allotted to a single yarn crossover in its initial (un-deformed) configuration is depicted in Fig. 1(a). It should be noted that the schematic displayed in Fig. 1(a) is a simplification of the corresponding unit-cell model based on the 3D fabric structure/architecture whose solid 8-node finite element discretization is depicted in Fig. 2(a). A comparison of the models depicted in Fig. 1(a) and 2(a) shows that the two yarns are simplified in Fig. 1(a) as two twomember truss elements each with a constant elliptical cross section. A deformed configuration of the simplified unit-cell model is depicted in Fig. 1(b).

The initial lengths of the corresponding single truss members are $L_{01}$ and $L_{02}$. It should be noted that due to the truss-character of the yarn elements, the contact between the yarns of the crossover is reduced to a point contact. Also, due to the finite thickness of the yarns, the yarn/yarn point contact corresponds to an initial-nonzero, $H_{01}+H_{02}$, distance between the summit points of the two-two-member truss elements, where $H_{01}$ and $H_{02}$ are the initial out-of-plane distances of the two summit points. Furthermore, it is assumed that, during deformation, the in-plane location of the contact point (i.e., the projection of the summit points to the shell surface) remains constant but the summit points can move in the out-of-plane direction.

When the unit cell is subjected to in-plane bi-axial normal strains, dimensions of the unit cell are changed from their initial values, $y_{01}$ and $y_{02}$ to $y_{1}\left(H_{1}\right)$ and $y_{2}\left(H_{2}\right)$, respectively. When the unit cell is stretched in a particular in-plane direction, the
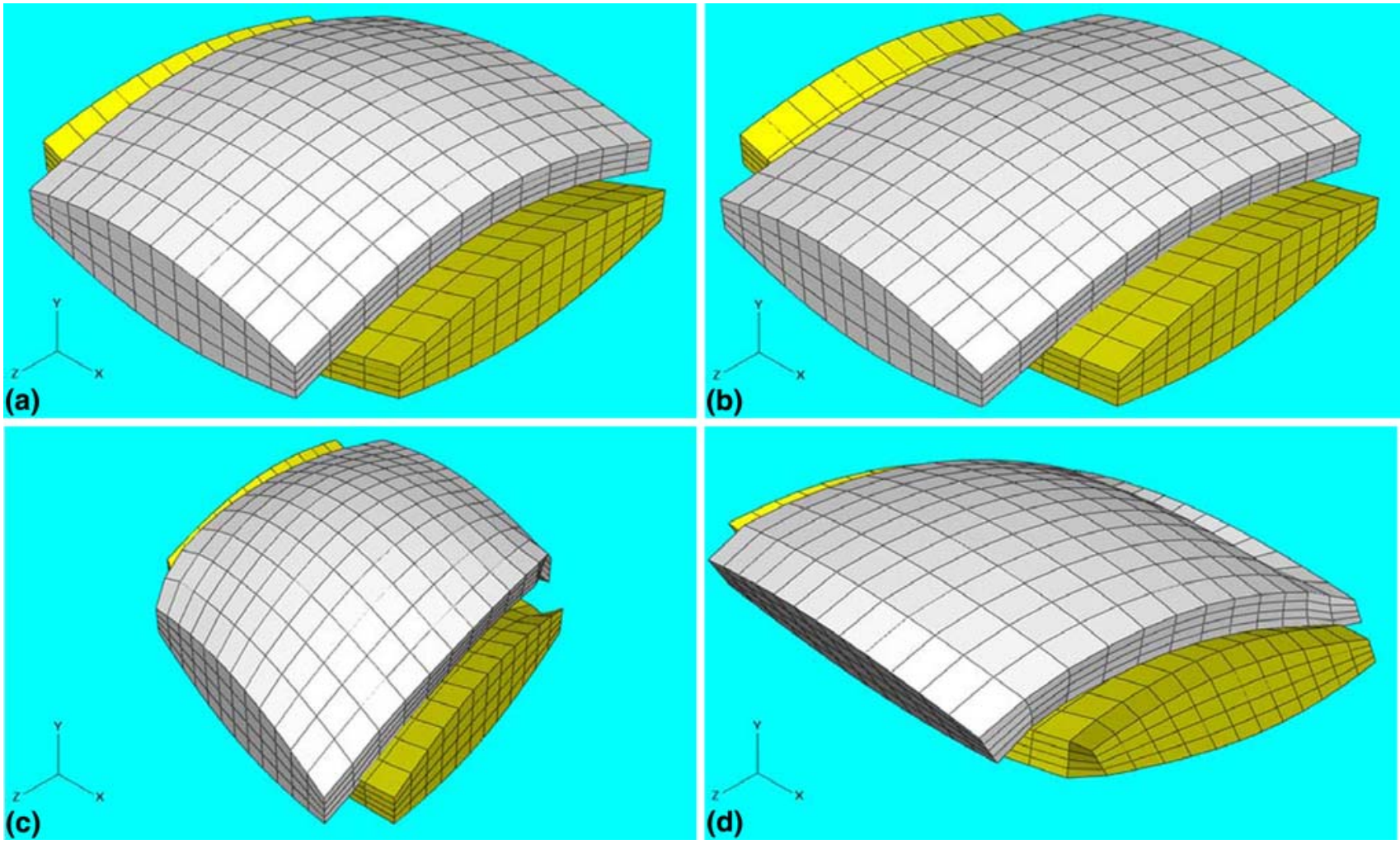

Fig. 2 Three-dimensional finite element representation of the unit cell in a plain-woven single-ply fabric: (a) initial configuration; (b) after inplane biaxial stretching; (c) after in-plane shear; and (d) after transverse shear 
corresponding yarn is either de-crimped/straightened or stretched. In the former case, no tension is built within the yarn, while in the latter case tension is created within the yarn and, if sufficiently high, can cause yarn failure. Also, the extent of tension in a given yarn is affected by the failure status of the other crossing yarn. If the crossing yarn is not broken and is in contact with the stretched yarn, tension will develop in the two yarns, the extent of which is dependent of the extent of contact over-closure (i.e., the reduction in distance of the two summit points relative to the initial distance) and the functional relationship which relates the contact over-closure with the magnitude of the contact force. Due to their low buckling resistance, compression cannot be built within the yarns when the cell is subjected to in-plane normal compressive strains.

\subsection{A Meso-Scale Unit-Cell Based Material Model for plain-Woven Single-Ply Fabric}

To restate, the main objective of the present work is to develop a meso-scale unit-cell based material model for a prototypical plain-woven single-ply Kevlar 129 fabric which can be used in large-scale computational analyses of multi-ply flexible-armor systems. The development of this model is presented in this section. Since the model is intended for the use in conjunction with shell finite elements, its development is divided in three parts: (a) the normal bi-axial in-plane response of the unit cell; (b) the in-plane shear response of the cell; and (c) the out-of-plane (transverse) shear response of the shell. In the following three sections, the development of each if these three components of the material model is presented. This is followed by a brief discussion of the implementation of the material model in a user material subroutine which is coupled with the commercial finite element program ABAQUS/Explicit (Ref 24).

As mentioned above, when the two yarns are not broken, in contact and the distance between the two summit points is smaller than the initial distance, contact force is developed between the two yarns and the extent of this force depends on the yarn-contact over-closure and a functional relationship which relates the contact-force magnitude to the contact overclosure. To determine the functional relationship between the yarn-contact over-closure $d_{\mathrm{c} 1}+d_{\mathrm{c} 2}=d_{\mathrm{c}}$ and the resulting contact force $F_{\mathrm{c} 1}$, a 3D solid FEM model of a single unit cell is constructed, Fig. 2(a) and subjected to the balanced bi-axial in-plane tensile loads and the total surface contact force monitored is a function of the yarn-contact over-closure. An example of deformed configuration of the unit cell is shown in Fig. 2(b) while the corresponding contact force versus yarn over-closure results obtained in this analysis are displayed in Fig. 3(a). Following Shahkarami and Vaziri (Ref 22), the $F_{\mathrm{c}}$ versus $d_{\mathrm{c}}$ relation is described using the following function:

$F_{\mathrm{c}}=\frac{b d_{\mathrm{c}}}{a-d_{\mathrm{c}}}$

It should be noted that Eq (1) ensures that the contact force initially increases linearly with the over-closure and as the overclosure begins to approach the yarn thickness, the contact force
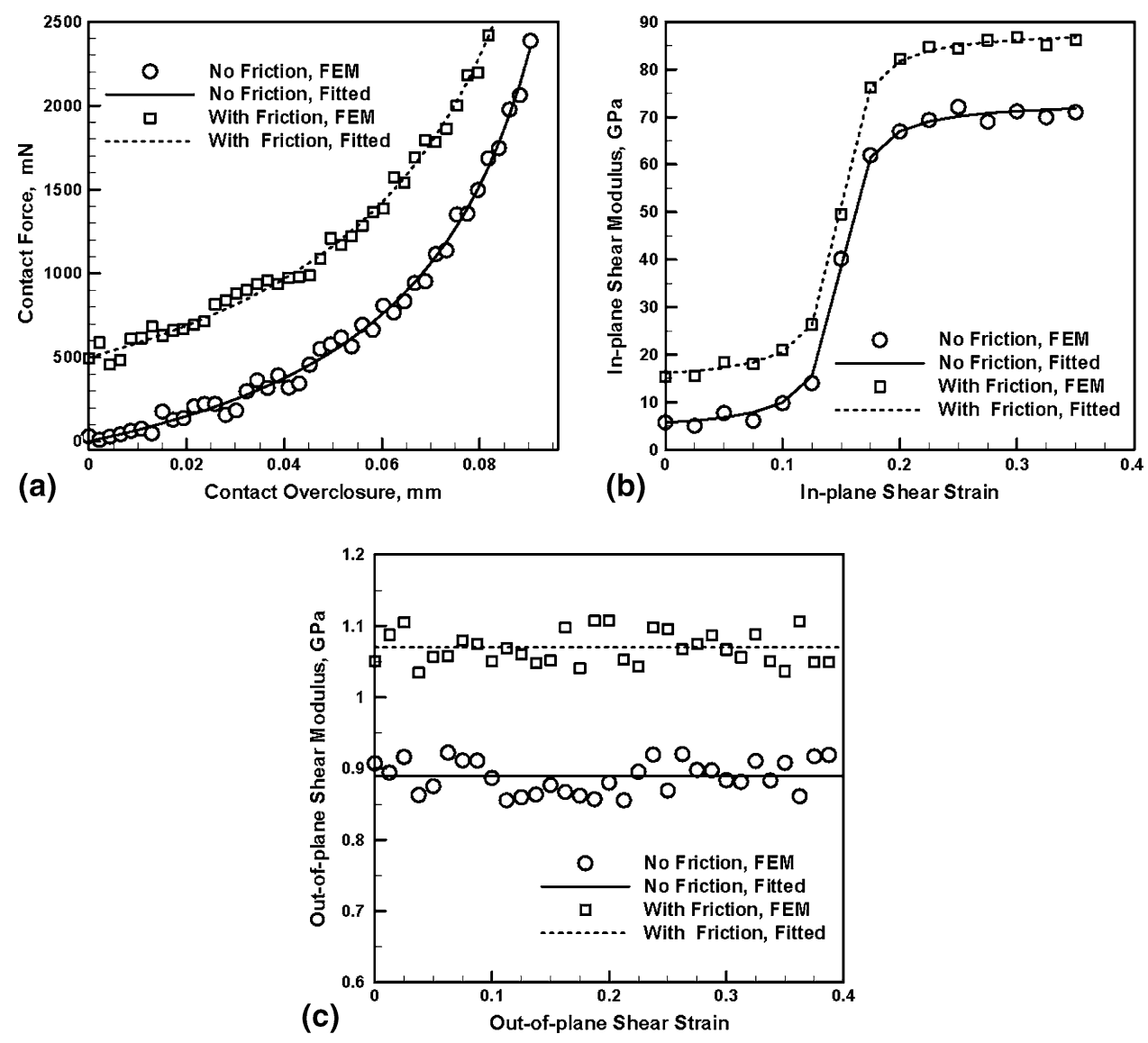

Fig. 3 Typical results obtained in 3D FEM analyses of the deformation of the unit cell: (a) under in-plane bi-axial tension; (b) in-plane shear; and (c) out-of-plane/transverse shear 
becomes excessively high. Using a simple unconstrained optimization procedure, the 3D solid FEM $F_{\mathrm{c}}$ versus $d_{\mathrm{c}}$ results displayed in Fig. 3(a) are fitted to the function defined by Eq (1) and the two parameters are determined as: $a=$ $0.12389 \mathrm{~mm}$ and $b=983.39 \mathrm{mN}$ in the absence of yarn/yarn friction and $a=0.12884 \mathrm{~mm}$ and $b=1298.1 \mathrm{mN}$ in the case of yarn/yarn frictional coefficient of 0.5 and these values for $a$ and $b$ are used in the remainder of the paper. It should be noted that the data pertaining to the case of yarn/yarn frictional coefficient of 0.5 in Fig. 3(a) are shifted upward by $500 \mathrm{mN}$ to improve clarity of the figure.

2.1.1 In-Plane Bi-Axial Behavior of the Unit-Cell. In the case of in-plane biaxial straining of the unit cell, several cases have to be considered depending on whether the strains are tensile or compressive and whether any of the yarns is broken:

(a) If both yarns are broken within a cell, the corresponding finite element is eroded, i.e. it is removed from the analysis;

(b) If one of the yarns is broken, then the other yarn is treated as being the only yarn in the unit cell. If the un-broken yarn is subjected to compression or the unitcell length in the direction of this yarn, $y_{i}(i=1$ or 2 and pertains to the un-broken yarn) is smaller than the corresponding initial yarn length $L_{0 i}$, then no load is supported by this yarn. In other words, zero in-plane normal stresses exist along the axis of both yarns. When $y_{i}>L_{0 i}$, then tension is developed within the un-broken yarn. This tension is defined by the following singleyarn expression as:

$T_{i}=E A_{i}\left(L_{i}-L_{0 i}\right) / L_{0 i}$

where $E$ is the yarn axial Young's modulus, $A_{i}$ is the crosssectional area, $L_{i}=y_{i}$ and $i$ refers to the unbroken yarn.

(c) If both yarns are intact, their respective unit-cell in-plane normal strains are both positive and there is a yarncontact over-closure, then the effect of the contact force has to be taken into account before the yarn tensions (and in turn, the corresponding in-plane unit-cell normal stresses) can be computed. A simple procedure for the calculation of the yarn tensions and unit-cell in-plane normal stresses is provided below. It should be noted that the total yarn-contact over-closure is defined as, i.e. $d c=d c_{1}+$ $d c_{2}$. Using single geometrical arguments and Fig. 1(a-b), the current yarn crimp heights can be defined as:

$H_{i}\left(d_{c i}\right)=H_{0 i}-d_{c i} \quad(i=1,2)$

In a same way, the current single-member truss-element lengths can be defined as:

$L_{i}\left(d_{c i}\right)=\sqrt{y_{i}^{2}+H_{i}^{2}} \quad(i=1,2)$

Equation (2) then defines the corresponding yarn tensions. Using the force equilibrium condition at the yarn summit points, the following expression can be derived for the corresponding contact forces:

$F_{c i}\left(d_{c i}\right)=2 T_{i}\left(d_{c i}\right) H_{i}\left(d_{c i}\right) / L_{i}\left(d_{c i}\right) \quad(i=1,2)$

The yarn/yarn forces at the two summit points, $F_{c 1}$ and $F_{c 2}$, defined by Eq (5) are equal to each other and are also equal to the contact force, $F_{\mathrm{c}}$, defined by Eq (1). Thus the following system of two nonlinear algebraic equations with two unknowns, $d_{c 1}$ and $d_{c 2}$, can be defined as:

$$
2 T_{1}\left(d_{\mathrm{c} 1}\right)\left(H_{1}\left(d_{\mathrm{c} 1}\right) / L_{1}\left(d_{\mathrm{c} 1}\right)\right)=2 T_{2}\left(d_{\mathrm{c} 2}\right)\left(H_{2}\left(d_{\mathrm{c} 2}\right) / L_{2}\left(d_{c 2}\right)\right)
$$

$2 T_{1}\left(d_{\mathrm{c} 1}\right)\left(H_{1}\left(d_{\mathrm{c} 1}\right) / L_{1}\left(d_{\mathrm{c} 1}\right)\right)=b\left(d_{\mathrm{c} 1}+d_{\mathrm{c} 2}\right) /\left(a-d_{\mathrm{c} 1}-d_{\mathrm{c} 2}\right)$

where $T_{i}\left(d_{\mathrm{c} i}\right), L_{i}\left(d_{\mathrm{c} i}\right)$ and $H_{i}\left(d_{\mathrm{c} i}\right)$ are defined by Eqs (2) to (4), respectively. Equations (6a) and (6b) can be readily solved using a Newton-Raphson routine. Once $d_{\mathrm{c} 1}$ and $d_{\mathrm{c} 2}$ are determined, the two yarn tensions can be computed using Eq (2) and in turn, the two in-plane unit-cell normal stresses can be determined as:

$\sigma_{i}=T_{i} / A_{i} \quad(i=1,2)$

(d) If both yarns are intact and one of the in-plane unit-cell normal strains is tensile while the other one is compressive, two possible cases can arise: (i) if the current summit-point distance is larger than its initial value $\left(H_{01}+H_{02}\right)$, then the yarns are not in contact and the yarn which is aligned in the compression-strain direction (the "compressed yarn") does not carry any load and the normal stress in the compression-strain direction is zero. As far as the other direction is concerned, tension can be developed in the yarn (when $y_{i}>L_{0 i}$, where $i$ pertains to the yarn aligned in the tensile-strain direction of the unit-cell, the "stretched yarn"). In this case, the yarn tension is given by the single-yarn tension relation, Eq (2). Otherwise, no tension is built within the yarn and the corresponding in-plane normal unit-cell stress also becomes zero; (ii) When yarn-contact over-closure is present, then tension builds up in both yarns and the two in-plane unit-cell normal stresses are both positive/ tensile. To determine the tensions in the two yarns, a similar procedure can be developed as the one used under point (c) in this section. The main difference is that the reference configurations from which the development of the yarn/yarn contact forces is measured are different in the two cases. In the case of biaxial stretching of the unit-cell, the reference configuration corresponds to the initial configuration of the fabric in which the two yarns are in contact but no over-closure (i.e., the contact force) is present. In the present case, it is advantageous to temporarily decouple the behavior of the two yarns. The summit-point height of the "compressed" yarn has increased under unit-cell compression. The summit-point of the "stretched" yarn is decreased due to unit-cell tension and the magnitude of this decrease is larger than the summit-point height increase of the "compressed" yarn. Hence, the reference configuration in this case corresponds to the one in which the decrease in the summit-point height of the "stretched" yarn is temporarily set equal to the summit-point height increase of the "compressed" yarn. Then, further decrease in the summit-point height of the "stretched" yarn will cause a further increase in the summit-point height of the "compressed" yarn which leads to a contact-force build-up and to the development of tension in both yarns. Once account is taken of the new reference configuration, yarn tensions and the corresponding unit-cell normal stresses can be calculated using the relations identical to those presented under point (c) in this section. 
2.1.2 In-Plane Shear Behavior of the Unit-Cell. It should be noted that the material model presented in the previous section provides a way for assessing only the in-plane normal stresses of the unit cell. Since no friction between the two-two-member truss elements is taken into account in Fig. 1, the unit-cell model could not be used to assess its in-plane shear behavior. To overcome this limitation, the 3D unit-cell finite element model displayed in Fig. 2(a) is subjected to an increase in-plane shear and the corresponding shear stress is determined by dividing the total in-plane shear force by the corresponding yarn cross-sectional area. An example of deformed configuration of the unit cell is displayed in Fig. 2(c) while the corresponding shear stress versus shear strain results obtained in this analysis are displayed in Fig. 3(b). The FEM results displayed in Fig. 3(b) are next fitted to an inverse tangent function, denoted by a solid line in Fig. 3(b), to obtain a relationship between the in-plane shear modulus, $G_{\text {in-plane, }}$ and the in-plane shear strain, $\varepsilon_{\text {in-plane. The following functional }}$ relations were obtained: $G_{\text {in-plane }}(\mathrm{GPa})=38.45+23.744 \mathrm{atan}$ $\left(67.0 \varepsilon_{\text {in-plane }}-26.8\right)$ in the absence of yarn/yarn friction, and $G_{\text {in-plane }}(\mathrm{GPa})=41.142+22.26 \mathrm{atan}\left(69.0 \varepsilon_{\text {in-plane }}-27.6\right)$ in the case of yarn/yarn frictional coefficient of 0.5 . It should be noted that the data pertaining to the case of yarn/yarn frictional coefficient of 0.5 in Fig. 3(b) are shifted upward by $10 \mathrm{GPa}$ to improve clarity of the figure.

2.1.3 Out-of-Plane (Transverse Shear) Behavior of the Unit-Cell. To determine the out-of-plane (transverse shear) behavior of the fabric, the 3D FEM model of the unit-cell displayed in Fig. 2(a) is subjected to an out-of-plane shear and the corresponding shear stress determined by dividing the applied transverse force by the yarn cross-sectional area. An example of deformed configuration of the unit cell is displayed in Fig. 2(d) while the corresponding transverse shear modulus versus transverse shear strain results obtained in this analysis are displayed in Fig. 3(c). The results displayed in Fig. 3(c) are fitted to a constant relation yielding the transverse shear moduli of 0.89 and $0.97 \mathrm{GPa}$ for the cases of yarn/yarn frictional coefficient of 0.0 and 0.5 , respectively. It should be noted that the data pertaining to the case of yarn/yarn frictional coefficient of 0.5 in Fig. 3(c) are shifted upward by $0.1 \mathrm{GPa}$ to improve clarity of the figure.

\subsection{Implementation of the Material Model in a User-Material Subroutine}

To enable the use of the newly derived meso-scale unit-cell based material model in computational analyses, the model is next implemented in the material user subroutine, VUMAT, of the commercial finite element program ABAQUS/Explicit (Ref 24). This subroutine is compiled and linked with the finite element solver and enables ABAQUS/Explicit to obtain the needed information regarding the state of the material and the material mechanical response during each time step, for each integration point of each element. In the present work, first-order 4-node general-purpose reduced-integration shell elements (ABAQUS/Explicit designation S4R) are used. Due to the use of the Simpson's numerical-integration method for the calculation of through-the-thickness deformation response of the shell, an odd number $(>1)$ of integration points has to be used in the through-the-thickness direction. The results obtained in the present work suggest that selecting three integration points provides a good compromise between computational efficiency and accuracy.
The coupling algorithm between the ABAQUS/Explicit finite element solver and the VUMAT Material User Subroutine at each time increment at each integration point of each element can be summarized as follows: (a) The corresponding previous time-increment stresses and material state variables as well as the current time-step strain increments are provided by the ABAQUS/Explicit finite element solver to the material subroutine. In the present work, the unit cell strains, warp and weft yarn summit-point heights, yarn lengths, as well as the flags pertaining to the failure status of the yarns and the deletion status of the element are used as state variables; and (b) using the information provided in (a), and the meso-scale unit-cell used material model presented in the previous section, the material stress state as well as values of the material state variables at the end of the time increment are determined within the VUMAT and returned to the ABAQUS/Explicit finite element solver. In addition, the changes in the total internal and the inelastic energies (where appropriate) are computed and returned to the solver.

One should note that within the VUMAT only the normal and shear in-plane responses of the shell material are computed. Transverse shear stiffness of the shell elements, as computed using the 3D FEM procedure outlined in the previous section, has to be defined as part of the overall FEM model definition outside the VUMAT. Using the provided values for the transverse shear modulus, ABAQUS/Explicit uses a simple procedure in which the transverse response of a shell is approximated by the transverse response of an analogous solid finite element.

To provide a more detailed insight into the implementation of the current meso-scale unit-cell based material model in the VUMAT, a flow chart is provided in Fig. 4. It is important to

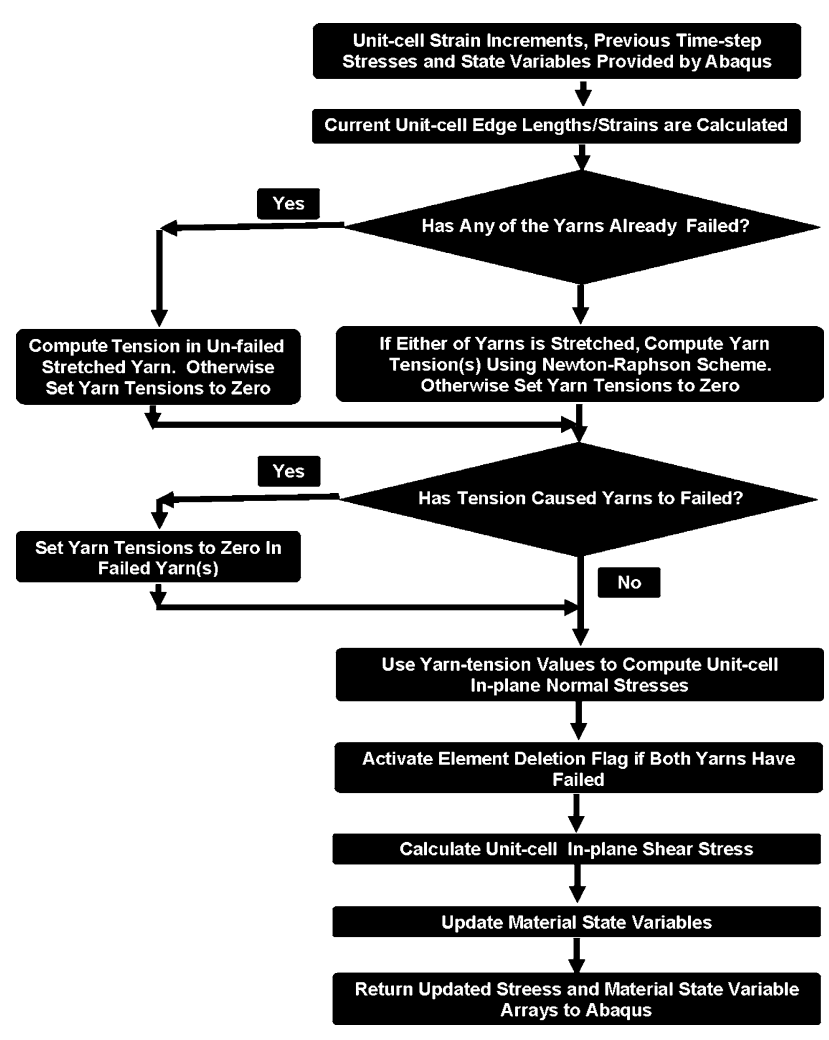

Fig. 4 The flow chart used during implementation of the mesoscale unit-cell based material model in a User-material subroutine 
note that ABAQUS/Explicit provides tensorial shear strain rather than the engineering shear strain increments. The procedure used to compute unit-cell stresses at the end of the current time step is clearly dependent on failure status of the two yarns at the end of the previous time step and on the current (tension versus compression) state of the unit cell edges. Yarns cannot support compressive loads and broken yarns cannot support tensile loads. In both cases, the associated in-plane normal stress components are zero.

\section{Validation of the Material Model}

In this section, a simple projectile/armor impact problem is described. The problem is used to carry out preliminary testing and validation of the meso-scale unit-cell based material model for plain-woven fabric developed in the present work. The initial configurations of the projectile/armor finite element systems analyzed here are shown as Fig. 5(a-b). In both cases, a rigid spherical projectile with a $4 \mathrm{~mm}$ radius and a $2.12 \mathrm{~g}$ weight is propelled at an initial velocity of $400 \mathrm{~m} / \mathrm{s}$ in the direction normal to the single-ply armor surface and toward the center-point of the armor. The armor is modeled as a $36 \mathrm{~mm}$ by $36 \mathrm{~mm}$ square patch. In Fig. 5(a), the plain-woven fabric structure is modeled explicitly by snaking through orthogonally oriented warp and weft yarns. The square-shaped fabric patch contained 44 warp and 44 weft yarns. Yarns are considered to have a constant $0.040 \mathrm{~mm}^{2}$ hexagonal cross-sectional area. The hexagonal cross section was used since this is the best approximation to the actual elliptical cross section which can be obtained using two-element wide yarns and the "Nodal Thickness" option available in ABAQUS/Explicit. The yarn width is set to $0.615 \mathrm{~mm}$, the peak height to $0.105 \mathrm{~mm}$, the crimp height to $0.088 \mathrm{~mm}$, and crimp wave-length to $1.64 \mathrm{~mm}$. All these values are consistent with those used by Duan et al. (Ref 10-14) and, for an effective yarn density of $0.6 \mathrm{~g} / \mathrm{cm}^{3}$, they yield an effective fabric areal-density of $3.31 \mathrm{~g} / \mathrm{cm}^{2}$.

Yarns are treated as a fiber-assembly homogenized material and represented using a linear-elastic orthotropic (or more precisely, transversely isotropic) material model with the unique material direction being aligned in the yarn-axis direction). The orthotropic linear-elastic yarn-material properties used are listed in Table 1 and they relate to the corresponding properties of Kevlar ${ }^{\circledR} 129$. Low values for the transverse normal and shear moduli and for the Poisson's ratios listed in Table 1 arise from the fact that the fibers bundled within yarns are only weakly coupled to each other.

In the case of Fig. 5(b), the armor is modeled as a constantthickness shell-based continuous surface. In other words, warp and weft yarns and their weaving are not modeled explicitly. The effect of yarn weaving, however, is included implicitly through the use of the meso-scale unit-cell based material model which is assigned to each shell element of the fabric in Fig. 5(b).

The effect of the following three different types of far-field boundary conditions applied to the edges of the fabric patch were considered: (a) all four fabric edges are clamped; (b) two opposite fabric edges are clamped and the other two are free; and (c) all four fabric edges are set free.

Yarn/yarn and projectile/fabric interactions are modeled using a simple penalty-based algorithm. A simple Coulomb model was used to account for yarn/yarn and projectile/fabric friction. Two frictional conditions were considered: (a) both the yarn/yarn friction coefficient $\mu_{\mathrm{y} / \mathrm{y}}$ and the projectile/fabric friction coefficient $\mu_{\mathrm{p} / \mathrm{f}}$ are set to 0.5 ; and (b) no yarn/yarn or projectile/fabric friction.

Due to explicit account of the warp and weft yarns and their weaving which entails the use of finer meshes, the FEM model displayed in Fig. 5(a) is typically computationally three to four times more expensive than the one displayed in Fig. 5(b).

As a part of the meso-scale unit-cell based material-model validation procedure, the interaction between the projectile and the armor is analyzed using the two FEM model and two

\section{Table 1 The orthotropic linear elastic material data} (GPa) for yarn (Ref 11)

\begin{tabular}{lllllllll}
\hline $\mathbf{E}_{\mathbf{1 1}}$ & $\mathbf{E}_{\mathbf{2 2}}$ & $\mathbf{E}_{\mathbf{3 3}}$ & $\mathbf{G}_{\mathbf{1 2}}$ & $\mathbf{G}_{\mathbf{1 3}}$ & $\mathbf{G}_{\mathbf{2 3}}$ & $\boldsymbol{v}_{\mathbf{1 2}}$ & $\boldsymbol{v}_{\mathbf{1 3}}$ & $\mathbf{v}_{\mathbf{2 3}}$ \\
\hline 164.0 & 3.28 & 3.28 & 3.28 & 3.28 & 3.28 & 0.0 & 0.0 & 0.0 \\
\hline
\end{tabular}

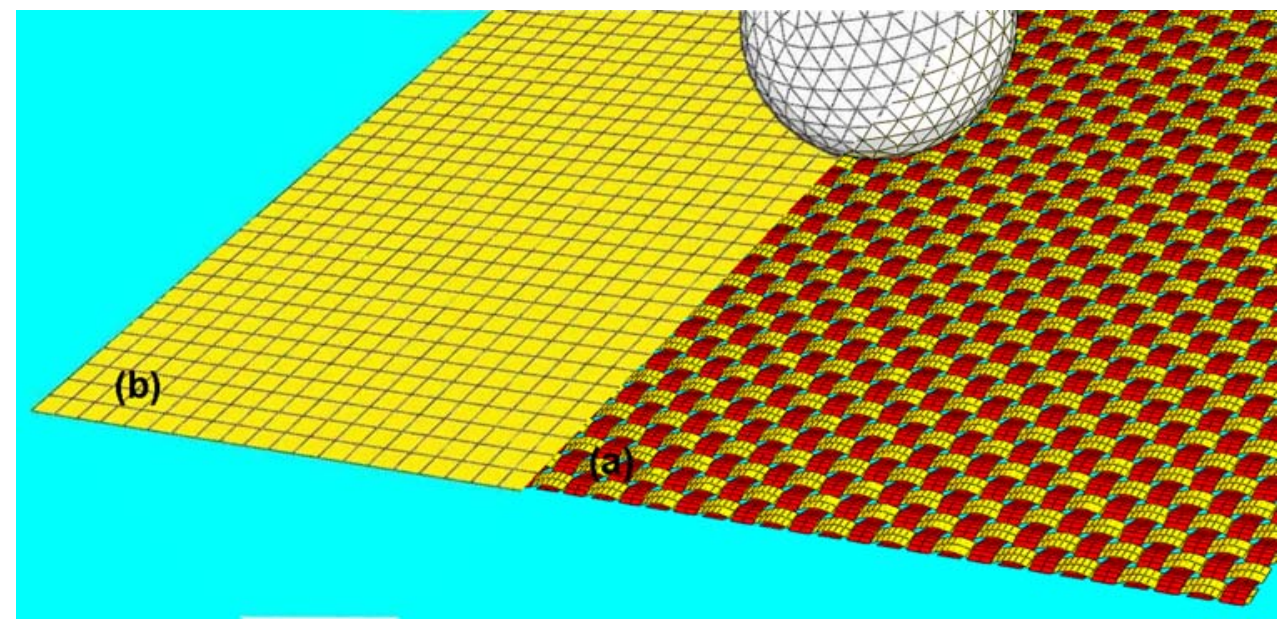

Fig. 5 The initial configurations of the projectile/fabric systems for (a) a shell FEM analysis in which the effects of yarn weaving are included implicitly through the use of an meso-scale unit-cell based material model; and (b) a shell FEM analysis in which yarn weaving is modeled explicitly 
aspects of these interactions are closely examined: (a) the residual velocity of the projectile after the projectile has successfully penetrated the armor and (b) the extent, the temporal evolution and the spatial distribution of fabric-armor damage.

It is important to note that the impact of a projectile with the fabric is associated with the initiation of several phenomena, the most important of which are: (a) a resisting force is exerted by the fabric onto the projectile which causes a reduction in the projectile velocity; (b) at the same time, the fabric is being deformed and accelerated; (c) strain waves generated in the impact region propagate along the yarns toward the fabric edges, where they are reflected. In the absence of any external force acting on the projectile/fabric system, the total energy of the system must be conserved. In general, the energy dissipation due to projectile deformation, fiber intermolecular friction, wind resistance, and acoustic losses are all assumed to be negligible. Consequently, any loss in projectile kinetic energy $\Delta E_{\mathrm{pk}}$ can be mainly attributed to the following three energyabsorbing mechanisms: yarn strain energy $E_{\mathrm{ys}}$, yarn kinetic energy $E_{\mathrm{yk}}$, and the energy lost due to frictional sliding $E_{\mathrm{f}}$. The energy conservation principle then requires that:

$\Delta E_{\mathrm{pk}}=E_{\mathrm{ys}}+E_{\mathrm{yk}}+E_{\mathrm{f}}$

Duan et al. (Ref 10-13) clearly showed that the loss of projectile kinetic energy $\Delta E_{\mathrm{pk}}$ is governed (through the three aforementioned energy-absorption mechanisms) by several factors such as the material properties of the constituent fibers, fabric structure, boundary conditions, projectile geometry, impact velocity, friction between the projectile and the fabric, and yarn-to-yarn and fiber-to-fiber friction within the fabric itself.

The overall decrease in projectile kinetic energy, $\Delta E_{\mathrm{pk}}$, is traditionally determined using ballistic-impact experiments in which only the projectile initial velocity $v_{i}$ and the residual velocity $v_{\mathrm{r}}$ are measured. The overall decrease in projectile kinetic energy, $\Delta E_{\mathrm{pk}}$, is then defined as:

$\Delta E_{\mathrm{pk}}=\frac{1}{2} m\left[v_{\mathrm{i}}^{2}-v_{\mathrm{r}}^{2}\right]$

where $m$ is the mass of the projectile. Recently, Starratt et al. (Ref 25) developed a ballistic-impact method for continuous measurement of projectile velocity $v(t)$. The loss of projectile kinetic energy as a function of time $t$ during impact is then defined as:

$\Delta E_{\mathrm{pk}}=\frac{1}{2} m\left[v_{\mathrm{i}}^{2}-v(t)^{2}\right]$

It should be noted, however, that while this experimental technique may greatly help improve our understanding of the ballistic-impact behavior of fabrics, many essential physical phenomena accompanying projectile/fabric interactions can only be obtained using numerical analyses and simulations like the ones used in the present work.

\section{Results and Discussion}

As mentioned earlier, the primary purpose of the present work was to develop, implement, and validate a computationally efficient, meso-scale unit-cell based material model for plain-woven single-ply fabric armor. Since the key functional requirement for an armor system is to absorb the kinetic energy carried by the projectile, a quantitative comparison of the results pertaining to the temporal evolution of the absorbed energies (through yarn deformation and fracture, fabric acceleration, and frictional-sliding based energy dissipation) obtained using the two FEM formulations discussed in the previous section is presented in the subsequent sections. The results obtained for the case of far-field boundary conditions corresponding to the all four fabric edges being clamped, and for the two frictional conditions are presented and discussed in the next section. The effects of the far-field boundary conditions are then discussed in the subsequent section.

\subsection{Four-Edges Clamped Far-Field Boundary Conditions}

Yarn/yarn friction and projectile/fabric friction are generally present in experimental and field tests of flexible-fabric armor, and as shown by Tan et al. (Ref 24) in a series of postimpact fabric-inspection studies, play an important role in absorbing the projectile kinetic energy. For example, in the presence of friction yarn-crossover domains near the impact region are found to be characterized by extensive fiber breakage, while reduced friction promotes slippage between the warp and weft yarns and typically the impact-induced fabric-perforation is smaller than the projectile size. Consequently, the first case analyzed here is the one in which both the yarn/yarn friction coefficient $\mu_{\mathrm{y} / \mathrm{y}}$ and the projectile/fabric friction coefficient $\mu_{\mathrm{p} / \mathrm{f}}$ are set to a nonzero $(=0.5)$ value.

4.1.1 Fabric Deformation and Yarn Fracture in the Presence of Friction. When a projectile hits the armor, two waves are generated: (a) a longitudinal wave which travels away from the point of impact along the principal yarns (the yarns directly hit by the projectile) and along those secondary yarns (the yarns not directly impacted by the projectile) which are interacting with the principal yarns. Propagation of the longitudinal wave outward from the point of impact enables a large fraction of the fabric armor to undergo deformation and, thus, absorb the kinetic energy of the projectile; and (b) a transverse wave which propagates outward from the point of impact at a velocity substantially lower than the sound speed and is responsible for stretching of the principal and the "engaged" secondary yarns. Since the sound speed is controlled by the yarn axial stiffness and density, and these two quantities are identical in the two FEM models, the temporal and spatial evolutions of the longitudinal-wave front are found to be quite comparable in the two models (the results not shown for brevity). The temporal evolution and the spatial distribution of the transverse-wave front are affected to a greater extent by yarn/yarn interactions. Therefore, a comparison of the transverse-deflection wave-front propagation results obtained using the two FEM analyses can be considered as a good validity test for the meso-scale unit-cell based material model developed in the present work.

Temporal evolutions of deformation within the fabric obtained using the FEM analysis involving discrete orthogonal yarns plain-woven into a single-ply fabric (referred to as the "yarn-level FEM analysis") and the FEM analyses based on the meso-scale unit-cell material model (referred to as the "unit-cell based FEM analysis") are displayed in Fig. 6(a-d) and $7(\mathrm{a}-\mathrm{d})$, respectively. In these figures, side and top views of the fabric along with superimposed contour plots of the transverse displacement (the displacement normal to the fabric surface) are shown. A simple comparison of the results 

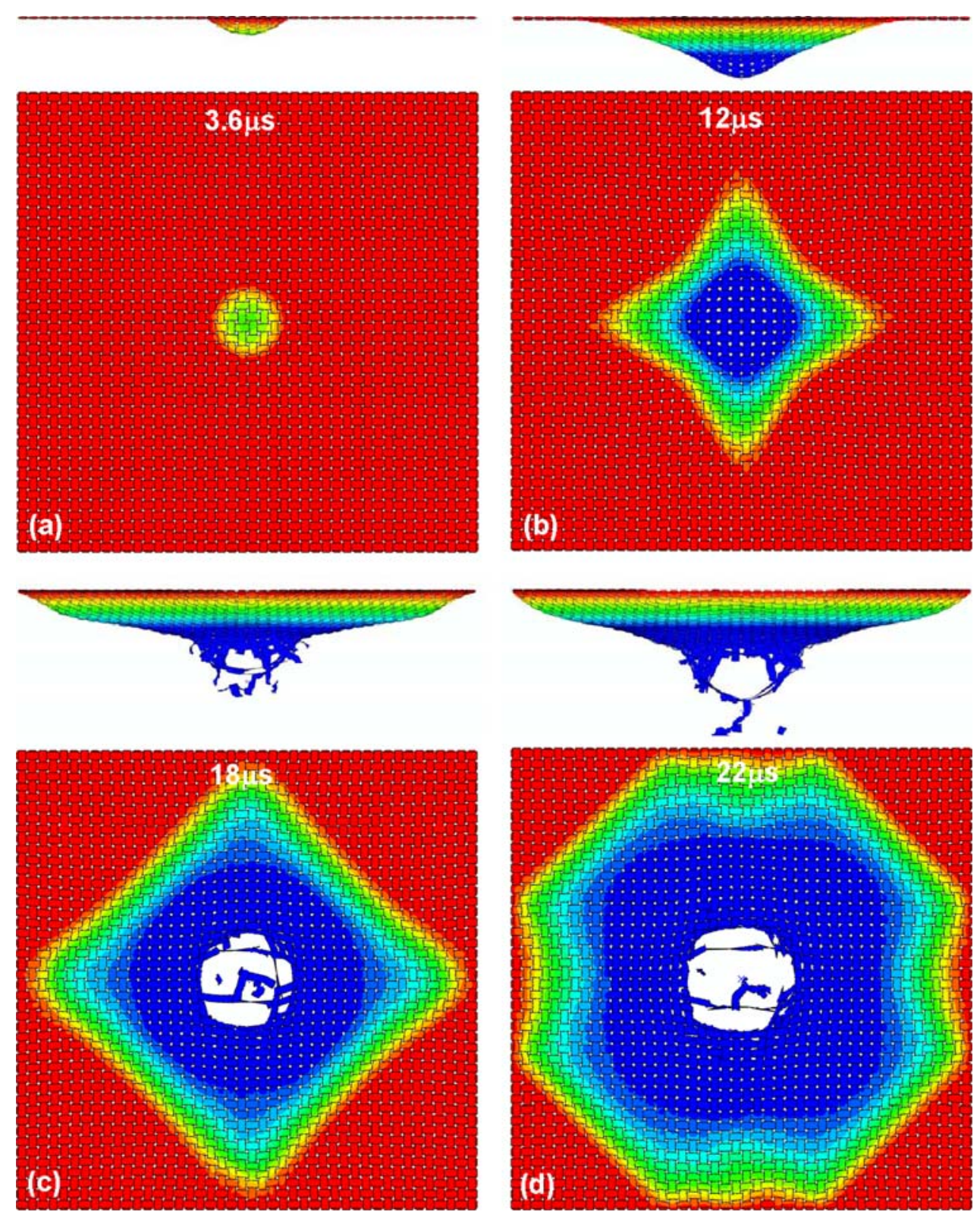

Fig. 6 The temporal evolution of deformation in the fabric for the yarn-level FEM model under the yarn/yarn $\mu_{\mathrm{y} / \mathrm{y}}$ and projectile/fabric $\mu_{\mathrm{p} / \mathrm{f}}$ friction coefficients of 0.5 . Contour bands correspond to different values of the transverse displacement, i.e. the displacements normal to the fabric surface. All four fabric edges are fixed

displayed in Fig. 6(a-d) and 7(a-d) reveals that the temporal evolution of the deformation state of fabric is quite similar in the two analyses and can be summarized as follows:

(a) The shape of the transverse-deflection wave-front in the fabric is initially nearly circular in both analyses and, thus, essentially identical to the shape of the projectile/ fabric contact-zone, Fig. 6(a) and 7(a);

(b) For the yarn-level FEM analysis, as the time proceeds, the transverse-deflection wave generated within the principal yarns (the yarns which are in direct contact with the projectile) propagates outward and, through their interactions with the secondary yarns (the yarns which are not in direct contact with the projectile), at the yarn crossovers, cause the secondary yarns to also deflect in the transverse direction. Consequently, the transversewave front begins to acquire a near square shape, with the square center coinciding with the impact-zone center, Fig. 6(b-c). In the case of the unit-cell based FEM analysis, the fabric is modeled as a continuous surface and, hence, the transverse wave can readily propagate in each radial direction from its source point (i.e., from the point of initial impact). Consequently, the transverse-deflection wave-front acquires a nearly circular (more precisely, a polygonal) shape, Fig. 7(b-c);

(c) In spite of the differences in the transverse-deflection wave front shape discussed in (b), the overall size of the fabric region which has undergone transverse deflections and the extents of these deflections are quite comparable in the two FEM analyses; 

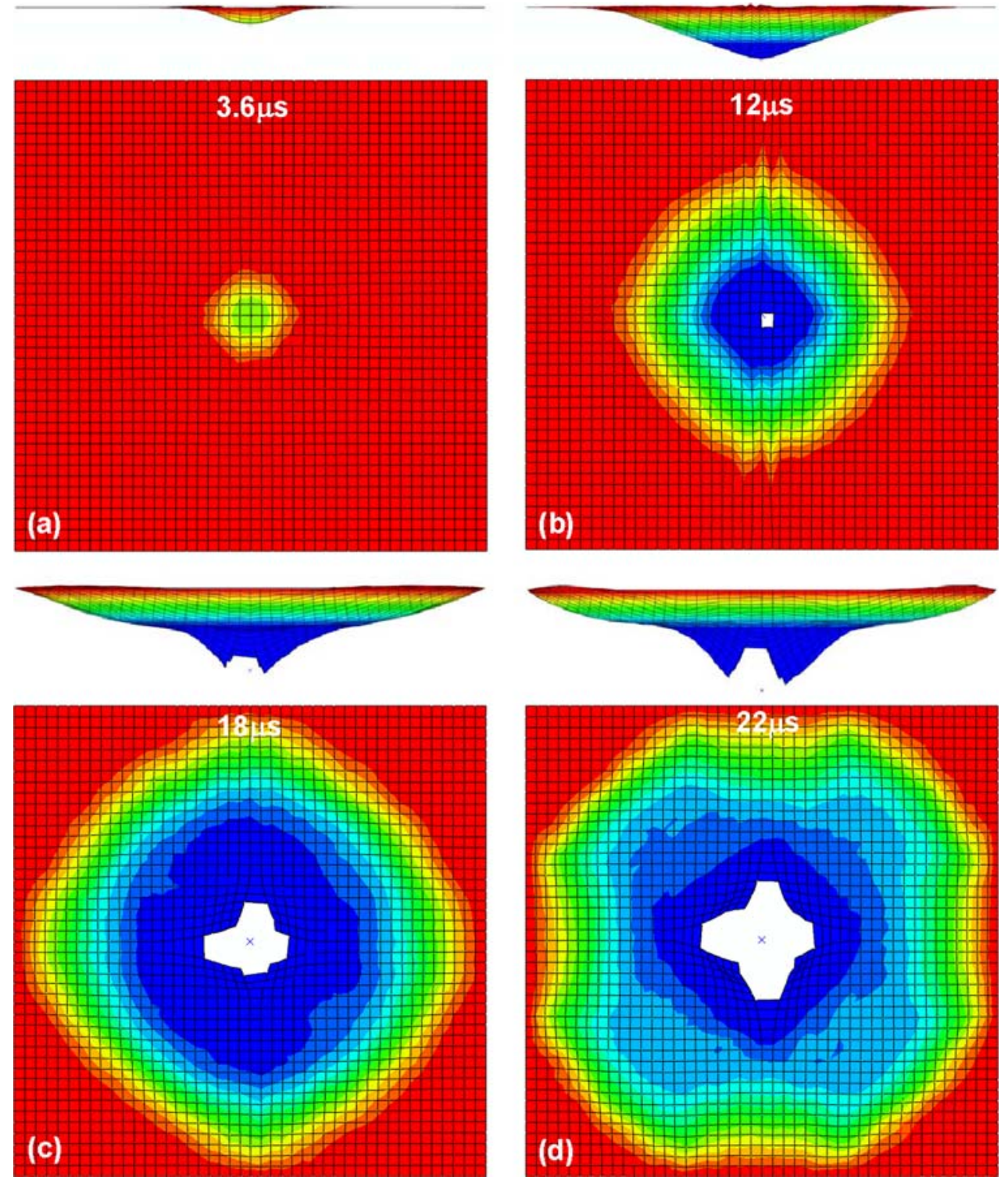

Fig. 7 The temporal evolution of deformation in the fabric for the unit-cell based FEM model under the yarn/yarn $\mu_{\mathrm{y} / \mathrm{y}}$ and projectile/fabric $\mu_{\mathrm{p} / \mathrm{f}}$ friction coefficients of 0.5 . Contour bands correspond to different values of the transverse displacement, i.e. the displacements normal to the fabric surface. All four fabric edges are fixed

(d) The (square-shaped) transverse-deflection wave-front continues to propagate toward the clamped edges of the fabric and, upon reaching the edges, it is reflected back toward the center of the impact zone and the wave front acquires an octagonal shape, Fig. 6(d). The more circleshaped transverse-deflection wave-front undergoes a similar shape change upon reaching and reflecting from the fabric edges, Fig. 7(d);

(e) Figure 7(b) reveals the first evidence of fabric failure for the case of the unit-cell based FEM analysis. At the same postimpact time, the yarns underneath the projectile in the case of the yarn-level FEM analysis undergo considerable lateral motion which reduces the extent of tension within the yarns and delays their failure, Fig. 6(b). However, at longer postimpact times, Fig. 6(c-d) and $7(\mathrm{c}-\mathrm{d})$, the extent of fabric damage becomes quite comparable in the two analyses; (f) The final size of the fabric penetration-hole (measured in the yarn directions) is somewhat smaller in the case of yarn-level analysis, Fig. 6(d), than in the unit-cell based case, Fig. 7(d). This relatively small discrepancy is related to the ability of some of the yarns which were pushed laterally by the projectile to recoil. Such elastic recovery was also observed in the case of the continuous fabric, but to lower extent. Nevertheless, the size of the penetration holes are quite comparable in the two cases and considering the fact that the FEM mesh is significantly finer and can capture more details in the case of the yarn-level FEM model, the overall agreement between the two analyses relative to the extent of damage in the armor can be deemed as reasonably good;

(g) At $\sim 25-27 \mu \mathrm{s}$, the projectile completely penetrates the fabric and continues to move at a residual velocity of $393.3 \mathrm{~m} / \mathrm{s}$ in the case of yarn-level FEM analysis and at 
a velocity of $393.7 \mathrm{~m} / \mathrm{s}$ in the case of unit-cell based FEM analysis; and

(h) One should note that despite the fact that the projectile/ fabric model has two vertical planes of symmetry, the damage region is asymmetric in both types of analyses. The reason for this is that the finite element discretization of the projectile into tetrahedron elements did not possess two planes of symmetry. The results displayed in Fig. 6(a-d) and 7(a-d) thus also reveal the effect of small geometrical perturbations in the (spherical) projectile on the fabric failure response.

4.1.2 Fabric Deformation and Yarn Fracture in the Absence of Friction. The discussion of the results displayed in Fig. 6(a-d) and 7(a-d) presented in the previous section clearly establish that, at the yarn/yarn and projectile/yarn friction-coefficient conditions of 0.5 , the two FEM analyses yield comparable results regarding the temporal evolution of the deformation within the fabric during impact. However, some minor discrepancies were observed relative to the extent of fabric damage. In this section, the corresponding computational results obtained/fabric friction are displayed in Fig. 8(a-d) and $9(\mathrm{a}-\mathrm{d})$, respectively. In these figures, side and top views of the fabric along with superimposed contour plots of the transverse displacement are shown. A simple comparison of the corresponding results reveals that the temporal evolutions of the deformation state of fabric are quite similar in the two FEM analyses at shorter postimpact times, Fig. 8(a-b) and 9(a-b), respectively. At longer postimpact times, Fig. 8(c-d) and 9(c-d), the agreement is less satisfactory. The main reason for the observed discrepancy is that in the yarn-level FEM analysis, the principal yarns can readily move sideways to avoid the projectile, while such yarn mobility is not present in the unitcell based FEM analysis. Consequently, less yarn breaking
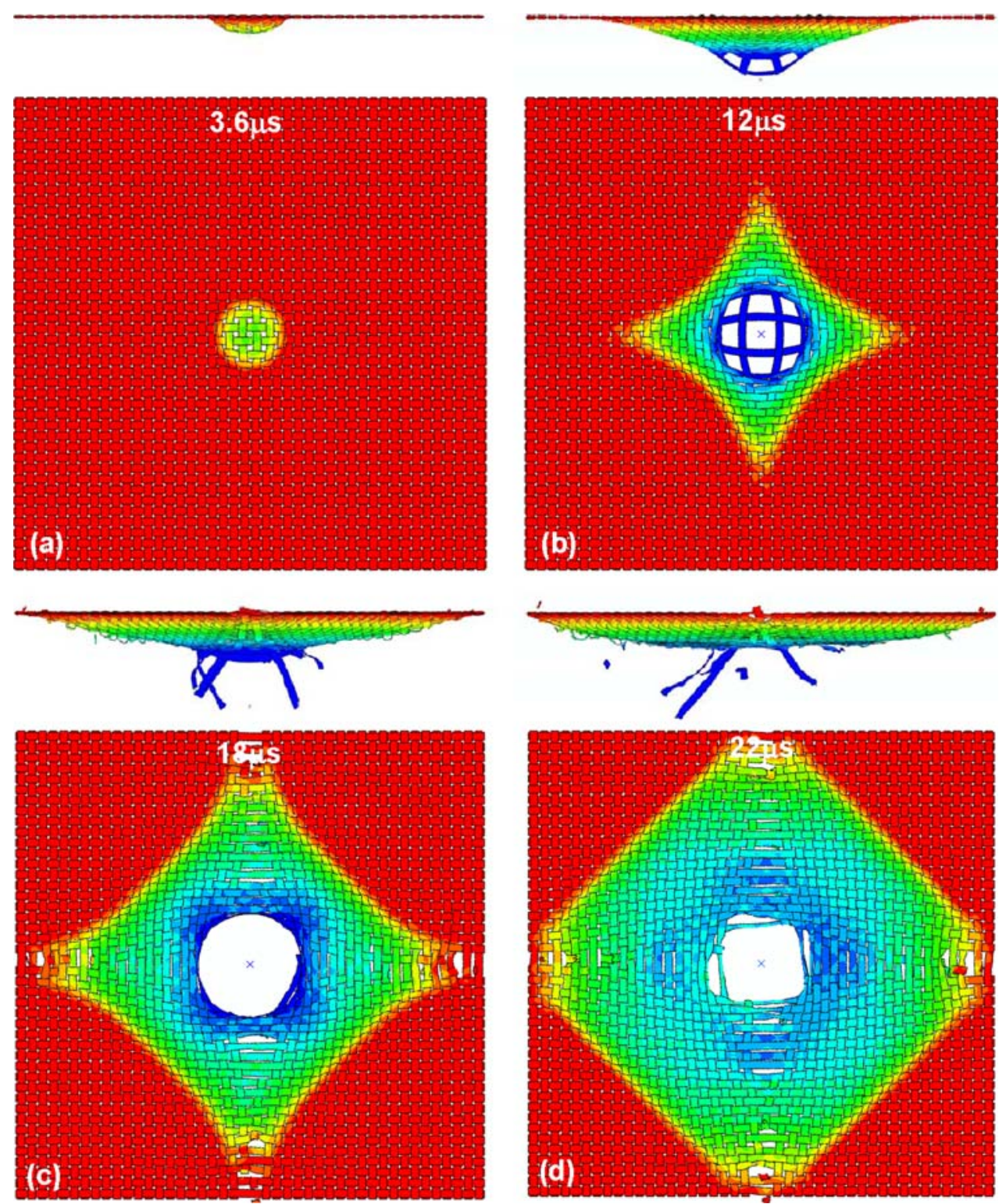

Fig. 8 The temporal evolution of deformation in the fabric for the yarn-level FEM model under the yarn/yarn $\mu_{\mathrm{y} / \mathrm{y}}$ and projectile/fabric $\mu_{\mathrm{p} / \mathrm{f}}$ friction coefficients of 0.0 . Contour bands correspond to different values of the transverse displacement, i.e. the displacements normal to the fabric surface. All four fabric edges are fixed 

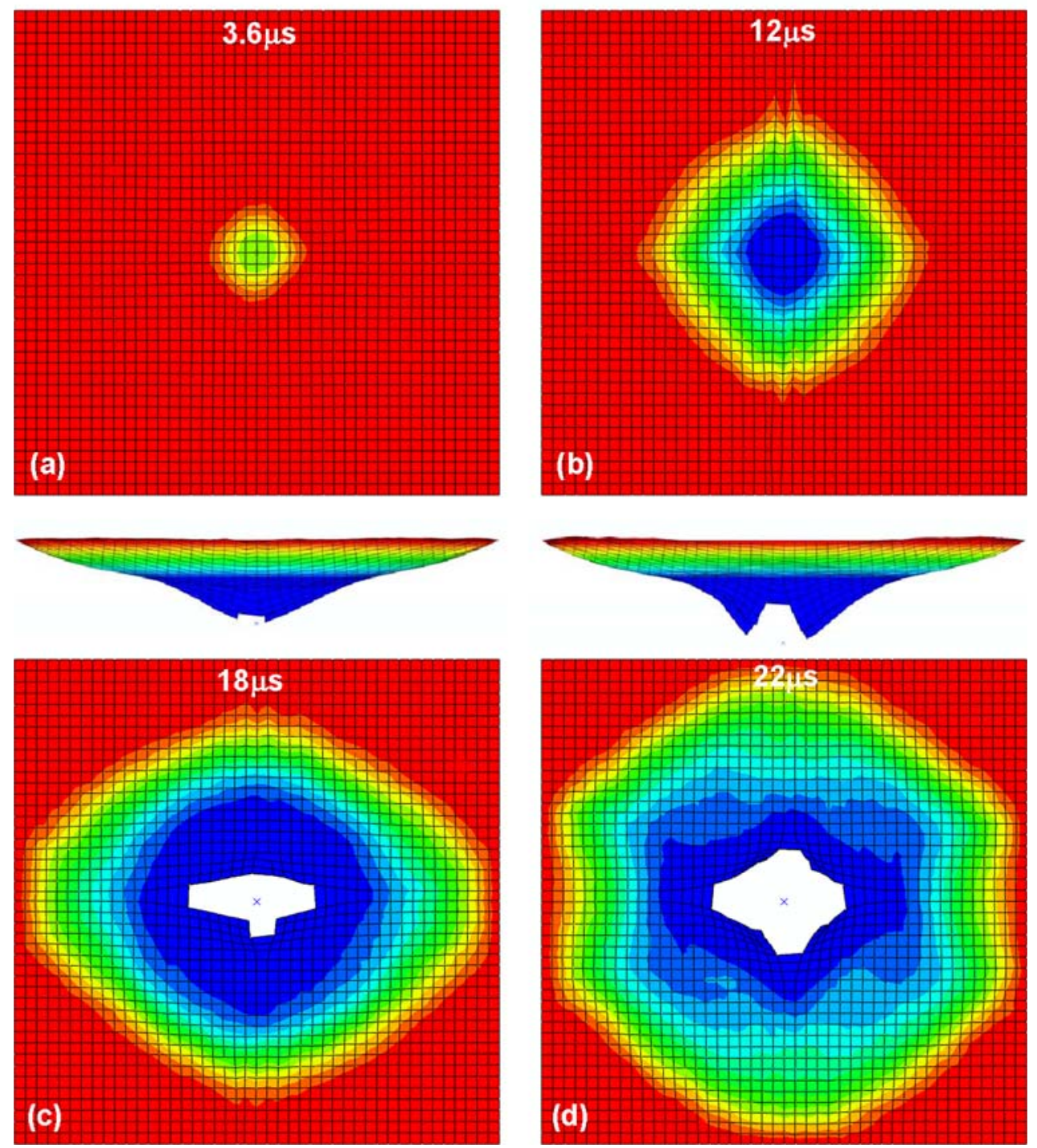

Fig. 9 The temporal evolution of deformation in the fabric for the unit-cell based FEM model under the yarn/yarn $\mu_{\mathrm{y} / \mathrm{y}}$ and projectile/fabric $\mu_{\mathrm{p} / \mathrm{f}}$ friction coefficients of 0.0 . Contour bands correspond to different values of the transverse displacement, i.e. the displacements normal to the fabric surface. All four fabric edges are fixed

takes place in the former case, and after the projectile has fully penetrated the armor, the "pushed aside" yarns tend to recoil. The yarn recoiling process reduces both the size of the penetration hole and the extent of residual lateral displacements in the fabric, Fig. 8(d) and 9(d), respectively. Despite these discrepancies, the overall final sizes of the penetration hole are comparable in the two analyses and, more importantly, the residual velocities of the projectile obtained in the two analyses $(398.6 \mathrm{~m} / \mathrm{s}$ in the case of the yarn-level and $397.8 \mathrm{~m} / \mathrm{s}$ in the case of the unit-cell based FEM analysis) are quite comparable.

As far as the effect of friction on the fabric deformation and failure behavior in the case of the yarn-level FEM analysis, Fig. 6(a-d) and $8(a-d)$, is concerned, the following main observations are made:

(a) Elimination of the yarn/yarn and the projectile/fabric friction; does not introduce initially significant changes in the temporal evolution and the spatial distribution of the transverse-deflection wave; and

(b) The deformation and the failure structure of the fabric in the impact region and in the nearby surrounding regions are greatly affected in the case of zero yarn/yarn and projectile/fabric friction conditions. Specifically, in the zero-friction case, yarns were substantially displaced in the in-plane directions away from the center of impact. This finding can be readily explained by the fact that the friction at the yarn crossovers provides resistance to the relative tangential motion of the yarns, while such resistance is absent in the zero-friction case. Consequently, in the zero-friction case yarns impacted by the projectile are pushed outward, a fewer number of yarns are broken and the projectile manages to penetrate the fabric mainly by "wedging" through it. 
A final examination of the corresponding results displayed in Fig. 8(a-d) and 9(a-d) suggests that, considering the fact that the FEM mesh is significantly finer and can capture more details in the case of the yarn-based FEM analyses, the overall agreement between the two analyses relative to the extent of deformation and damage in the armor can be deemed as less satisfactory than in the case when friction is present at the yarn/ yarn crossovers and projectile/fabric contact surfaces, Fig. 6(a-d) and 7(a-d). The results displayed in Fig. 6-9 were obtained under the projectile velocity of $400 \mathrm{~m} / \mathrm{s}$. A similar level of agreement between the corresponding results obtained in the two FEM analyses was obtained when the projectile velocity was doubled or halved relative to this value.

4.1.3 Fabric's Energy Absorption Potential. The results pertaining to temporal evolution of the three main energyabsorbing mechanisms (i.e., yarn strain energy, yarn kinetic energy, and frictional-sliding losses) are compared for the two types of FEM analysis under the two types of frictional conditions are presented and discussed in this section. The corresponding results for the two types of FEM analyses are displayed in Fig. 10(a-b) and 11(a-b), respectively. In Fig. 10(a-d) and 11(a-d), the quantities displayed along the vertical axis are all normalized by the corresponding maximum projectile energy loss. Also, the residual projectile velocities are indicated and their comparison suggests a quite good agreement between the corresponding values obtained using the two analyses. A simple comparison of the results displayed in Fig. 10(a-b) and 11(a-b) reveals that:

(a) A somewhat larger contribution of the frictional-sliding loss is present in the case of the yarn-level based analysis, Fig. 10(a) versus 11(a). This finding is consistent with the fact that, in the case of the unit-cell based analyses, yarn/yarn friction is not included explicitly and does not contribute to the frictional-sliding loss;

(b) The yarn strain-energy contribution for both friction conditions is higher in the case of the unit-cell based analysis. While this is expected considering the fact that the effect of yarn/yarn friction is included implicitly in the unit-cell based model through the friction-dependent in-plane shear term, the same argument cannot be made in the zero-friction case. Thus, the unit-cell based analysis tends to somewhat over-predict the strain-energy contribution;

(c) In the case of the yarn-level FEM analysis, the fullpenetration time (defined as a time when the projectile reaches its final velocity) is greater $(\sim 26 \mu \mathrm{s})$ in the presence of friction, Fig. 10(a), than $(\sim 15 \mu \mathrm{s})$ in the zerofriction case, Fig. 10(b). This finding is related to the fact that in the absence of friction the projectile can wedge through the fabric, while in the presence of friction more yarn failure is required before the projectile can reach its residual velocity. In the case of the unitcell based FEM analysis, the presence/absence of friction has less effect on the penetration time since no "wedge through" effect can take place, Fig. 11(a-b);

(d) The contribution of the frictional sliding mechanism to energy absorption is typically small. However, the presence of friction itself promotes projectile's kineticenergy absorption and, thus, increases fabric energyabsorption capacity by yarn straining and yarn acceleration. This finding is based on a comparison between the residual projectile velocities in the friction case,
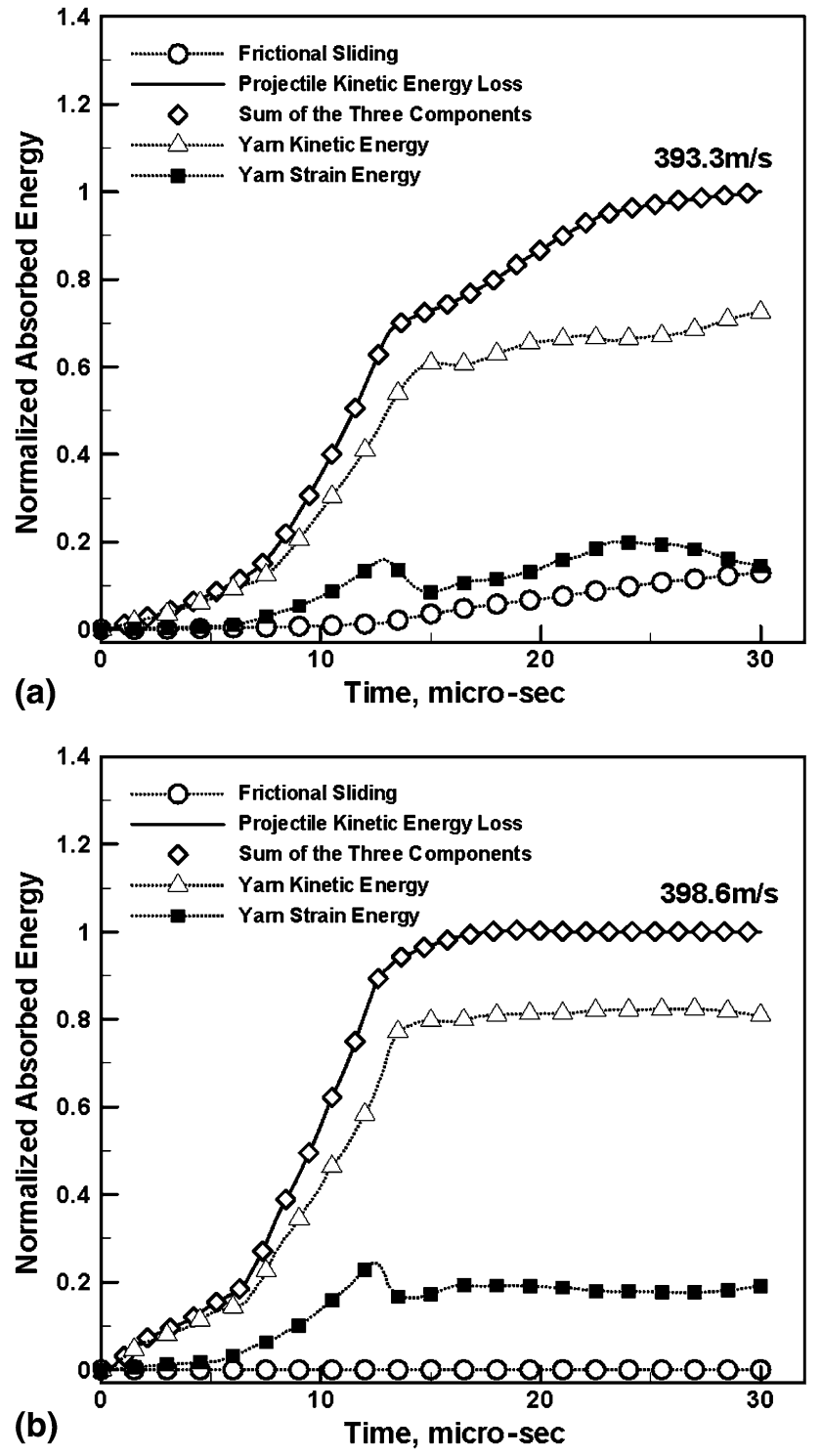

Fig. 10 The effect of yarn/yarn $\mu_{\mathrm{y} / \mathrm{y}}$ and projectile/fabric $\mu_{\mathrm{p} / \mathrm{f}}$ friction coefficients on the temporal evolution of projectile's kineticenergy loss and on different energy-absorbing mechanisms in the fabric obtained using the yarn-level FEM model: (a) $\mu_{\mathrm{y} / \mathrm{y}}=\mu_{\mathrm{p} / \mathrm{f}}=0.5$ and (b) $\mu_{\mathrm{y} / \mathrm{y}}=\mu_{\mathrm{p} / \mathrm{f}}=0.0$

Fig. 10(a) and 11(a) and in the zero-friction case, Fig. 10(b) and 11(b);

(e) Once the projectile begins to interact with the fabric, the contributions of yarn straining to the energy-absorption process increases. As the projectile starts to penetrate the armor, further yarn straining either ceases or increases at a significantly lower rate. After the armor is fully penetrated, the fabric kinetic energy component of the total yarn energy experiences a relative increase (due to yarn recoiling) and likewise the frictional-sliding component of the total yarn energy increases; and

(f) The level of agreement between the two FEM analyses regarding the energy absorption capacity of armor under the projectile velocity of $400 \mathrm{~m} / \mathrm{s}$ as seen in Fig. 10(a-b) and $11(\mathrm{a}-\mathrm{b})$ was also observed under different initial projectile velocities. 

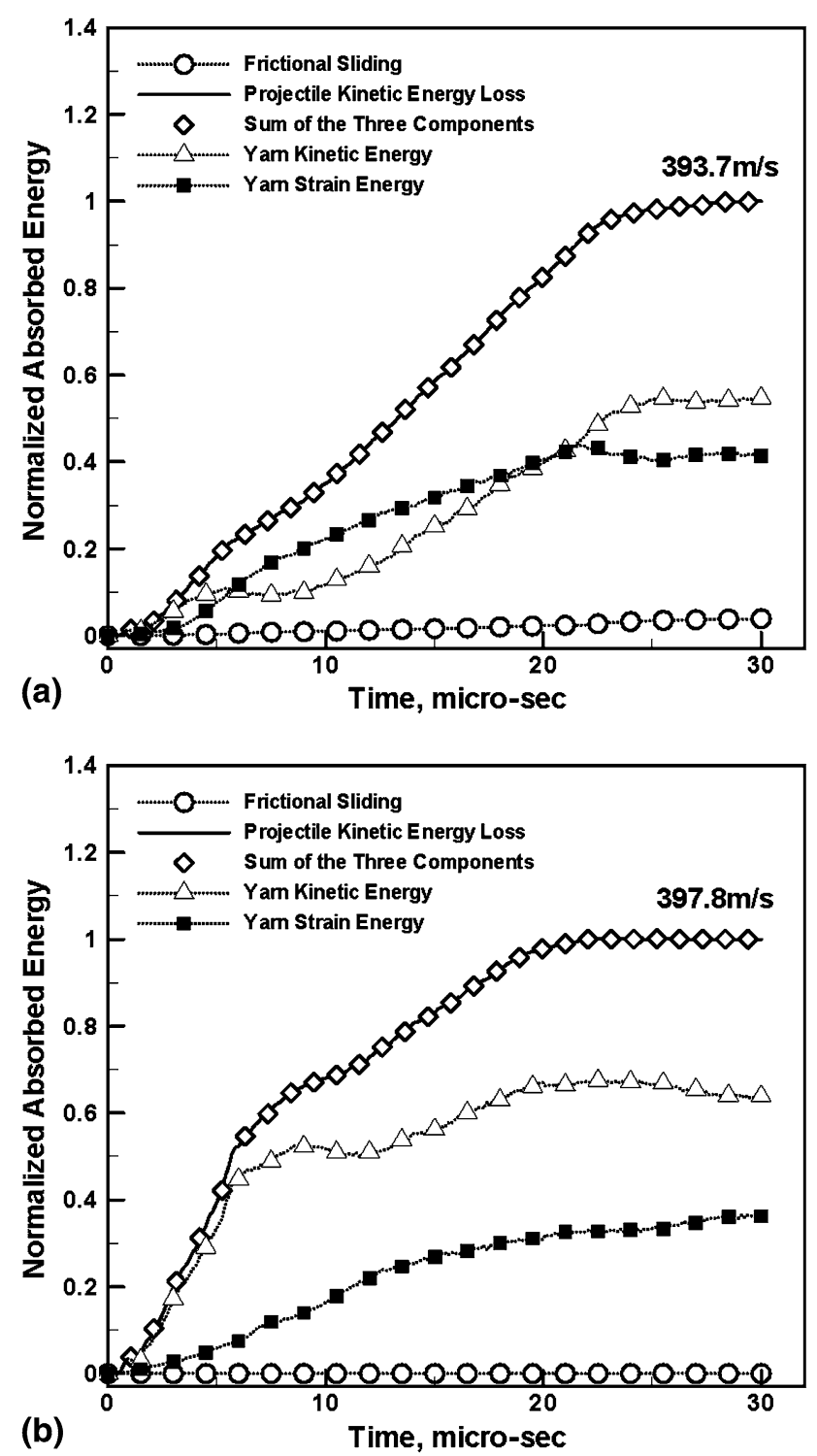

Fig. 11 The effect of yarn/yarn $\mu_{\mathrm{y} / \mathrm{y}}$ and projectile/fabric $\mu_{\mathrm{p} / \mathrm{f}}$ friction coefficients on the temporal evolution of projectile's kineticenergy loss and on different energy-absorbing mechanisms in the fabric obtained using the unit-cell based FEM model: (a) $\mu_{\mathrm{y} / \mathrm{y}}=$ $\mu_{\mathrm{p} / \mathrm{f}}=0.5$ and (b) $\mu_{\mathrm{y} / \mathrm{y}}=\mu_{\mathrm{p} / \mathrm{f}}=0.0$

\subsection{The Effect of Far-Field Boundary Conditions}

It is generally found that the nature (free versus fixed) of boundary conditions affects the ballistic response of the armor and its ability to absorb kinetic energy as well as it affects the relative contributions of various energy-absorbing mechanisms (Ref 10-13). The results presented and discussed in Section 4.1 show that the meso-scale unit-cell based material model can reasonably well account for the deformation/failure behavior and the energy-absorbing capacity of a plain-woven single-ply fabric under fixed boundary conditions being applied to all four fabric edges. In this section, a comparison is made between the corresponding results obtained using the yarn-level FEM and unit-cell based FEM analyses under other boundary conditions. Due to space limitations, the results analogous to those displayed in Fig. 6-11, but for the boundary conditions corresponding to: (a) two free (opposite) and two clamped (opposite) fabric edges, and (b) four free fabric edges will not be shown in this section. Instead these results will be discussed and related to their counter parts presented in the previous section.

As far as the spatial distribution and temporal evolution of the transverse displacements within the fabric are concerned, similar observations were made as those related to Fig. 6-9. The main differences were observed near the edges of the fabric along which the boundary conditions were changed from clamped to free. Specifically, when the transverse-wave front reaches the free fabric edges, the edges begin to be pulled-in toward the point of impact resulting in "bowed-in" edges. This situation is similar to the one observed in sheet-metal forming where reduction in the clamping force may lead to a transition from stretch-forming to deep-drawing. As a result of this transition, in the present case the largest maximum transverse deflections were obtained under the four-free edge boundary conditions.

The overall agreement between the extents of fabric damage predicted by the two FEM analyses under the two types of boundary conditions analyzed in this section is at a level similar to that observed in the previous section.

As far as the energy-absorbing capacity of the fabric is concerned, it is observed that this capacity is increased when the boundary conditions applied along the edges of the fabric are changed from clamped to free. This observation is consistent with the fact that free boundary conditions lower the rate of stress increase in the fabric and, thus, delays fabric failure. Consequently, the fabric is given longer time to interact with the projectile and absorb its kinetic energy before penetration.

\section{Application of the Material Model in the Analysis of Personnel Protection}

\subsection{Problem Definition}

A bullet-resistant vest typically consists of a front and a rear flexible mat, both made of multiple cross-stitched layers of ballistic fabric and housed within a carrier made of conventional garment material. According to the National Institute of Justice (NIJ) specification "Ballistic Resistance of Personal Body Armor NIJ Standard-0101.04," the highest level of protection offered by soft body armor is level III-A. Additional protection is attained in vests with front and rear pockets for the accommodation of ceramic, metallic, or high-performance polymer-fiber "rifle" plates. In the present work, the material model reviewed in the previous section has been utilized to carry out a series of computational analyses aimed at assessing the ballistic performance of (rifle-plate free) soft body armor employing Kevlar ${ }^{\odot} 129$ based flexible mats. These mats consist of multiple stitched fabric layers interlaced with thin plastic films for added flexibility.

The results obtained in the previous section clearly revealed that the ballistic performance of a single-layer fabric is greatly affected by the nature of the boundary conditions as well as by the presence/absence and stiffness/compliance of a backing support structure. Consequently, to properly assess the response of the protective system, a simple model of a representative upper torso was introduced as the backing support structure. The effect of the fabric carrier was modeled implicitly through the use of distributed pressure loading over the surface of the 
fabric plies. The ballistic performance was assessed against a 9-mm full metal jacketed (FMJ) round nose bullet with a nominal mass of $8.0 \mathrm{~g}$ and fired at an initial velocity of $332 \mathrm{~m} / \mathrm{s}$ (a NIJ level II-A threat). Typical exploded and detailed views of the bullet/vest/upper-torso model in its initial configuration are displayed in Fig. 12(a-b), respectively.

To represent the human body compliance, the upper torso is modeled as a deformable shell structure. In addition, four Cartesian and four Cardan connectors with nonlinear elastic and damping behavior are inserted between four nodes around the sternum area and four nodes belonging to the back of the upper torso. The effect of the lower torso is accounted for by adding one mass element and one rotary-inertia element which were coupled with the lower edge of the upper torso. The center $160 \mathrm{~mm}$ by $160 \mathrm{~mm}$ chest region which may experience the largest effect of the bullet strike is meshed using $2 \mathrm{~mm}$ square elements. The rest of the upper chest is meshed using coarser three- and four-node elements with a typical edge length of 5-7 mm. To obtain seamless connection between the two regions, a set of multi-point kinematic constrains are applied along the boundary separating the two regions. Typically, the upper torso contained around 9000 elements. The elastic material properties for the shell elements of the upper torso and the connector properties were taken from Ref 26 .

The soft-body flexible chest mat is also modeled using shell elements. However, the mat was divided into three sections: (a) the innermost 20-layer thick $50 \mathrm{~mm}$ by $50 \mathrm{~mm}$ square section containing the meso-scale unit-cell based material model developed in the present work; (b) a $100 \mathrm{~mm}$ by $100 \mathrm{~mm}$ outer square frame containing only the elastic portion of the fabric model used in the innermost region. Regions (a) and (b) are meshed using $1 \mathrm{~mm}$ square elements; and (c) a coarsely meshed single-layer outer region containing three- and four-node elements with a typical edge length of 5-7 mm. Typically, the chest mat contained 220,000 elements. The outer region was filled with the same elastic material as region (b). Again, to ensure continuity, multi-point kinematic constraints were applied along the boundaries separating the finely meshed and the coarsely meshed regions. In addition, the outer boundary of the $100 \mathrm{~mm}$ by $100 \mathrm{~m}$ region is assumed to

(a)
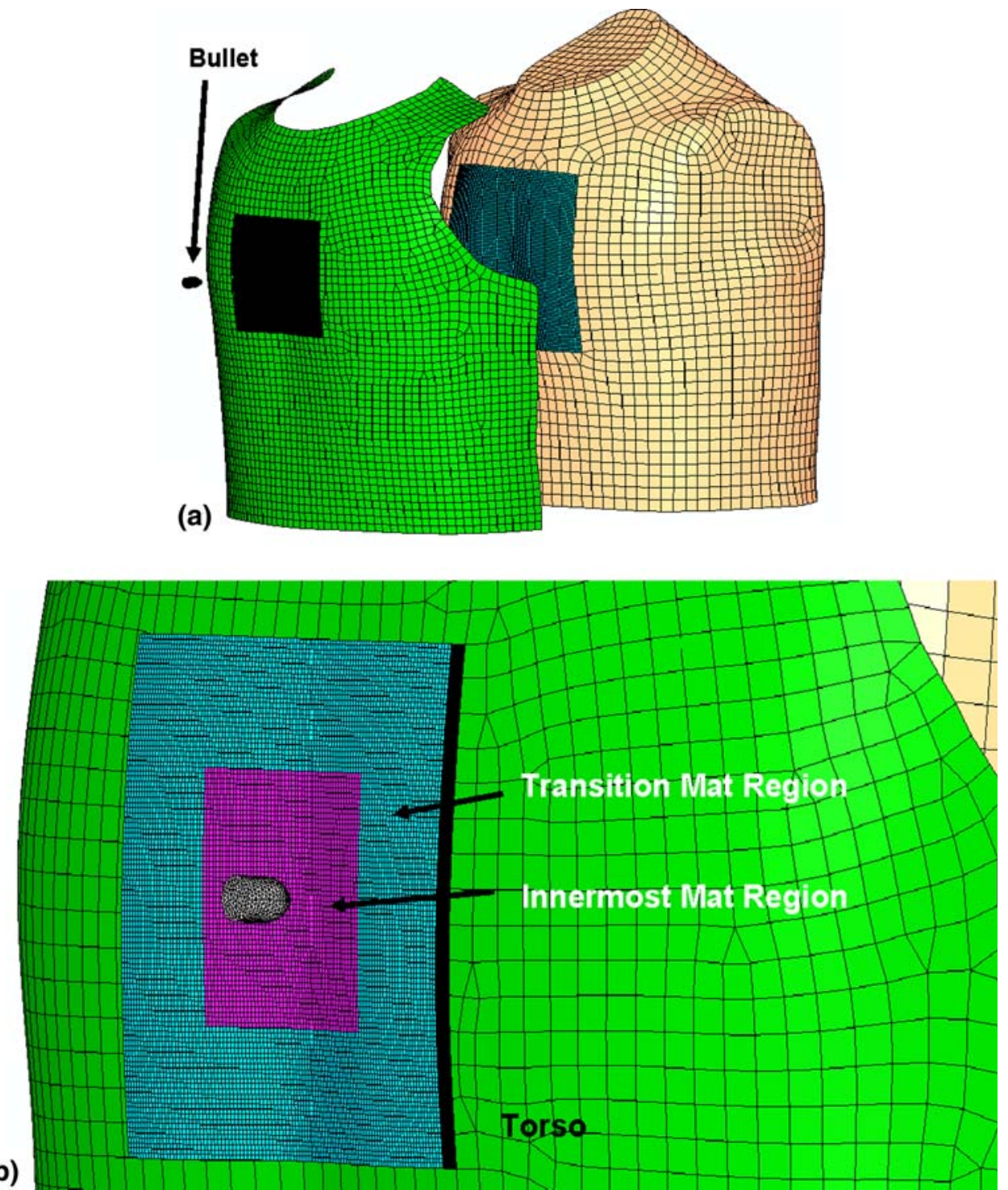

Fig. 12 The initial configuration of the bullet/chest mat/upper-torso model: (a) an exploded view; and (b) a detailed view of the impact region 
coincide with the cross-stitching lines in the mat. To mimic the effect of cross stitching, multipoint kinematic constraints were also applied to the corresponding ("stacked") nodes of all the layers through the thickness of the mat. The thin plastic sheets separating adjacent layers in the mat were not modeled explicitly. Rather, their effect was included implicitly by adjusting the value of the inter-layer friction coefficient.

The bullet was meshed using tetrahedron elements with an average edge length of $1 \mathrm{~mm}$. Typically, the copper jacket region contained 3000 elements while the lead core region contained 3000 elements. The jacket and the core share nodes along their contact surface, i.e. a perfect jacket/core interfacial bonding is assumed. Details regarding the material models for copper and lead based on a linear equation of state, the Johnson-Cook strength model, the Johnson-Cook failure model, and an erosion algorithm based on the maximum allowable instantaneous geometrical strain used in the present work can be found in Ref 27.

Interactions between the projectile, armor mat layers, and torso were modeled using a "Hard Contact Pair" type of contact algorithm. Within this algorithm, contact pressures between two bodies are not transmitted unless the nodes on the "slave surface" contact the "master surface." No penetration/ over-closure is allowed and there is no limit to the magnitude of the contact pressure that could be transmitted when the surfaces are in contact. Transmission of shear stresses across the contact interfaces is defined in terms of a static, $\mu_{\mathrm{st}}$, and a kinematic, $\mu_{\text {kin }}$, friction coefficient and an upper-bound shear stress limit, $\tau_{\text {slip }}$ (a maximum value of shear stress which can be transmitted before the contacting surfaces begin to slide).

To prevent hourglassing effects which may arise due to the use of reduced-integration elements, a default value of hourglass stiffness was used. No mass-scaling algorithm was used to increase the maximum stable time increment.

Computational analyses were run on a machine with two $2.33 \mathrm{GHz}$ Quad-core Intel Xeon processors with $16 \mathrm{~GB}$ of RAM. A typical computational analysis run to the point when either the bullet is defeated of the soft body-armor mat is fully penetrated required between 2 and $3 \mathrm{~h}$ of (wall-clock) time.

\subsection{Representative Results}

A bullet-resistant vest not only has to stop a bullet, but also has to prevent excessive intrusion of its back layers into the wearer's body at the point of impact. Otherwise, localization of the impact to a small area may cause severe internal injuries. To achieve this function, soft body armor must be capable of mitigating blunt trauma by distributing the localized impact loading over a large portion of the torso. The ability of the flexible protection to fulfill these functions is investigated in the present work. Specifically, the computational analysis described in Section 4.1 and the material model overviewed in Section 3 enabled investigations of: (a) the energy-absorbing capacity of the personnel protection system needed to stop a projectile; and (b) the ability of personnel protection system to minimize blunt trauma caused by excessive intrusion of its back layers into the wearer's torso and highly localized impact loads. Since this portion of the work is in its early stages, only a few representative results will be shown and discussed in this section. However, the long-term goal of this work is to develop computational capabilities which will enable full-scale design, optimization, and performance validation of personnel protection systems.
A set of examples of the results obtained in this portion of the present work is displayed in Fig. 13-16. The final deformed shape of the defeated $9-\mathrm{mm}$-caliber round nose projectile is displayed in Fig. 13. Plastic deformation of the projectile has been found to be a significant energy-absorbing mechanism

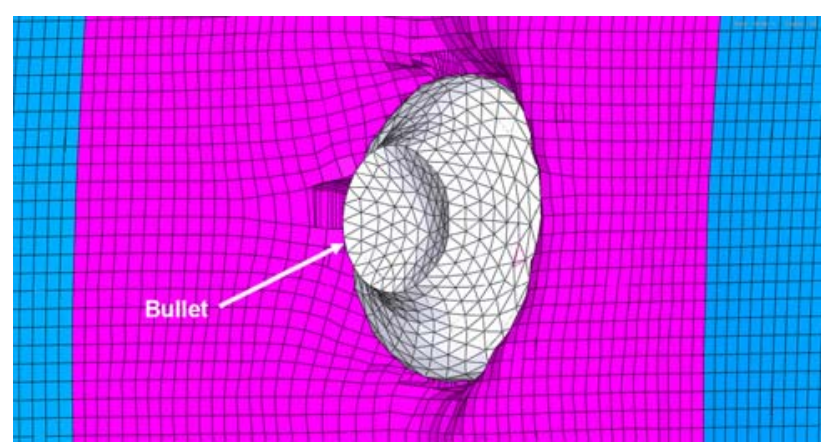

Fig. 13 Final deformed shape of a defeated $9 \mathrm{~mm}$ round-nose bullet

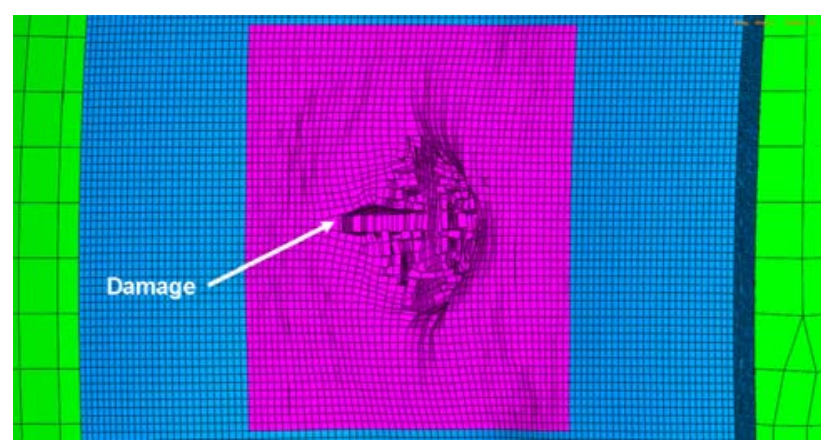

Fig. 14 Spatial distribution of the postimpact damage within the soft body armor mat

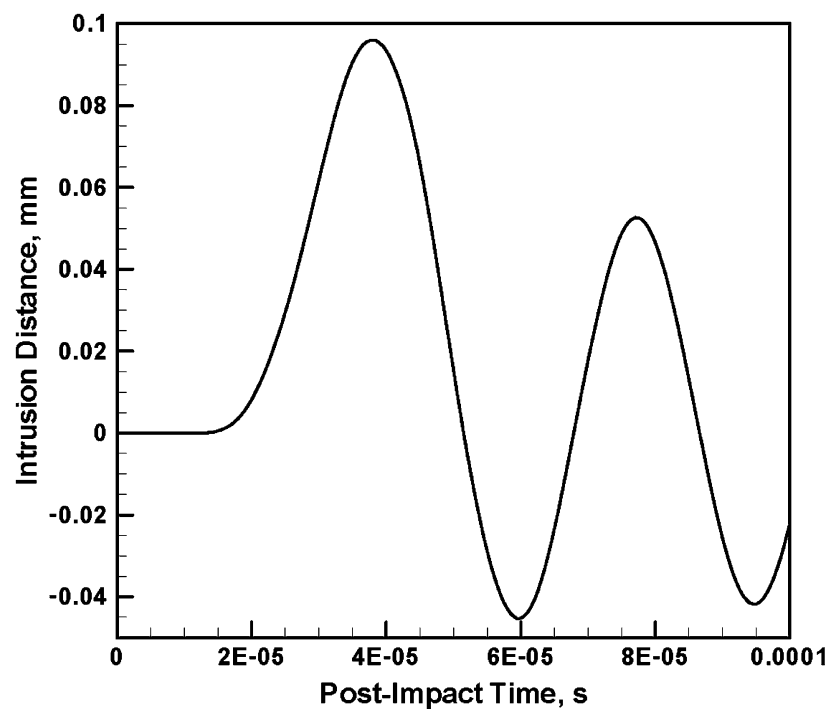

Fig. 15 Temporal evolution of the torso intrusion by the back layer of the vest 


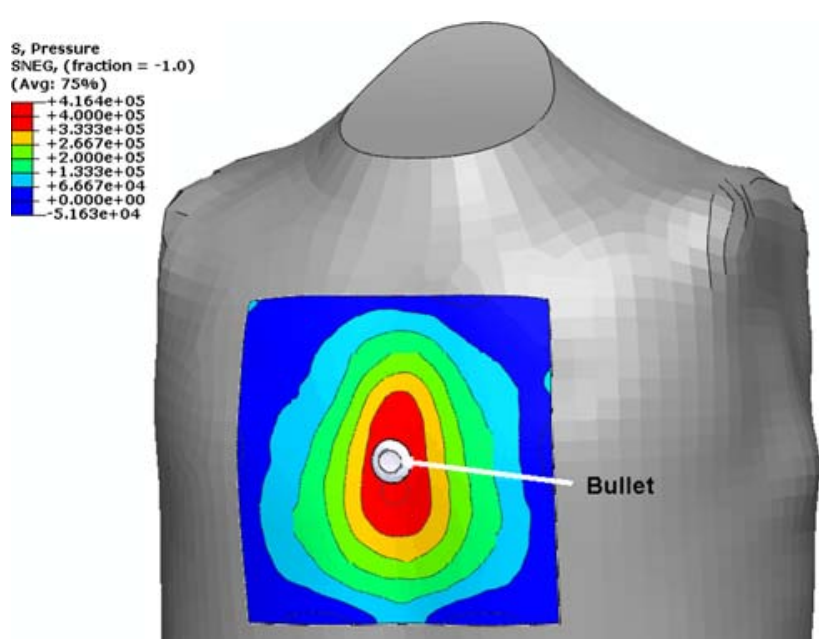

Fig. 16 Spatial distribution of the surface pressure corresponding to the maximum torso intrusion

capable of absorbing between 10 and $20 \%$ of the projectile kinetic energy. The partially penetrated soft-armor mat region has been displayed in Fig. 14. It is generally found that buildup of the strain energy and acquisition of the kinetic energy by different layers of the soft-armor mat both act as important mechanisms for absorption of the projectile kinetic energy. The absolute magnitudes of these absorbed-energy components is found to be significantly affected by the extent of projectile/ mat-layer and inter-layer friction in a way similar to that described in Sect. 3. A more comprehensive investigation of the role of friction is underway.

Temporal evolution of the $x$-component of displacement of a node in the center portion of the sternum (a measure of the chest intrusion) is displayed in Fig. 15. The positive $x$-direction coincides with the direction of projectile motion. The maximum chest intrusion is found to be significantly affected by cross stitching of the flexible vest. This finding was subsequently related to a cross-stitching induced increase in soft body armor mat transverse stiffness. It should be noted that in the present formulation the behavior of the torso is nearly perfectly elastic. In other words, energy dissipation is achieved only through frictional losses at the torso/vest interface and within the four sternum connectors. This affects the results displayed in Fig. 15 in at least 2 ways: (a) the maximum intrusion is quite small (ca. $0.09 \mathrm{~mm}$ ); and (b) the intrusion is reversible and oscillatory. A more advanced model for the torso will be used in our future work.

The distribution of surface pressures corresponding to the maximum torso-intrusion condition is displayed in Fig. 16. It should be noted that, for improved clarity, the armor vest is not displayed in Fig. 16 and that the results displayed correspond to a case when the torso was rigidized (except for the $160 \mathrm{~mm}$ by $160 \mathrm{~mm}$ center region). As explained earlier, spreading of the impact load over a wider portion of the chest is an important functional requirement of soft body armor. The results displayed in Fig. 16 clearly show that impact loads are indeed spread over a wide region of the sternum. This can be seen by comparing the defeated projectile (displayed in Fig. 16) with the size of the region of the sternum subject to considerable surface loads.

As discussed earlier, application of the current computational procedure and material model to analyze the ballistic protection performance of soft body armor is in its early stages. Nevertheless, the preliminary results are encouraging and suggest that the procedure can be brought to the level that largescale design, optimization and performance validation of bodyarmor vests is feasible. The multi-disciplinary optimization framework needed has been recently developed by the present authors in Ref 28 will be utilized in our future work.

\section{Summary and Conclusions}

Based on the material-model development procedure utilized and the results of the subsequent computational analyses, the following main summary remarks and conclusions can be drawn:

1. A simple meso-scale unit-cell based material-model for plain-woven single-ply fabric armor is developed and implemented in a User-material Subroutine suitable for use with commercial explicit finite element programs.

2. The User-material Subroutine is coupled with the ABAQUS/Explicit finite element program and used in a series of transient nonlinear dynamic analyses of a plainwoven single-ply fabric armor impact by a spherical steel projectile in order to test and validate the material model.

3. By comparing the results obtained in a finite element analysis in which the User-material Subroutine is used with those obtained in a computationally more-costly FEM model in which yarn weaving is accounted for explicitly, it is found that the present meso-scale unit-cell material model for plain-woven single-ply fabric, under fixed boundary conditions applied to all four edges of the fabric, can reasonably well account for the observed temporal evolution and spatial distribution of transverse waves and damage within the armor and for the roles of various energyabsorbing mechanisms. Similar observations were made where different combinations of clamped and free boundary conditions were applied along the edges of the fabric.

4. To demonstrate the full utility of the present model, the model is used in a full scale computational investigation of impact into a personnel protection system chest mat placed in contact with a flexible structure mimicking a human torso.

\section{Acknowledgments}

The material presented in this paper is based on work supported by the U.S. Army/Clemson University Cooperative Agreements W911NF-04-2-0024 and W911NF-06-2-0042. The authors are indebted to Dr. Fred Stanton for the support and a continuing interest in the present work.

\section{References}

1. R.E. Wittman and R.F. Rolsten, Armor of Men and Aircraft, 12th National SAMPE Symposium, SAMPE, 1967

2. The Interceptor System, US Marine Corps, (http://www.marines. mil/marinelink/image1.nsf/lookup/200532317129?opendocument). Accessed 2 December 2008

3. D. Roylance and S.S. Wang, Penetration Mechanics of Textile Structures, Ballistic Materials and Penetration Mechanics, Amsterdam, Elsevier, 1980, p 273-292 
4. V.P.W. Shim, C.T. Lim, and K.J. Foo, Dynamic Mechanical Properties of Fabric Armour, Int. J. Impact Eng., 2001, 25, p 1-15

5. C.T. Lim, V.P.W. Shim, and Y.H. Ng, Finite Element Modeling of the Ballistic Impact of Fabric Armor, Int. J. Impact Eng., 2003, 28, p 13-31

6. A. Shahkarami, R. Vaziri, A. Poursartip, and K. Williams, A Numerical Investigation of the Effect of Projectile Mass on the Energy Absorption of Fabric Panels Subjected to Ballistic Impact, Proceedings of the 20th International Symposium on Ballistics, 2002, p 802-809

7. G.R. Johnson, S.R. Beissel, and P.M. Cunniff, A Computational Model for Fabrics Subjected to Ballistic Impact, Proceedings of the 18th International Symposium on Ballistics, Vol. 2, 1999, p 962-969

8. H.H. Billon and D.J. Robinson, Models for the Ballistic Impact of Fabric Armour, Int. J. Impact Eng., 2001, 25, p 411-422

9. D.A. Shockey, D.C. Erlich, and J.W. Simons, Improved Barriers to Turbine Engine Fragments, US Department of Transportation, SRI International, Menlo Park, CA, 2000

10. Y. Duan, M. Keefe, T.A. Bogetti, and B.A. Cheeseman, Modeling Friction Effects on the Ballistic Impact Behavior of a Single-Ply Highstrength Fabric, Int. J. Impact Eng., 2005, 31, p 996-1012

11. Y. Duan, M. Keefe, T.A. Bogetti, and B.A. Cheeseman, Modeling the Role of Friction During Ballistic Impact of a High-Strength PlainWeave Fabric, Int. Comp. Struct., 2005, 68, p 331-337

12. Y. Duan, M. Keefe, T.A. Bogetti, B.A. Cheeseman, and B. Powers, A Numerical Investigation of the Influence of Friction on Energy Absorption by a High-Strength Fabric Subjected to Ballistic Impact, Int. J. Impact Eng., 2006, 32, p 1299-1312

13. Y. Duan, M. Keefe, T.A. Bogetti, and B. Powers, Finite Element Modeling of Transverse Impact on a Ballistic Fabric, Int. J. Mech. Sci., 2006, 48, p 33-43

14. G.M. Zhang, R.C. Batra, and J. Zheng, Effect of Frame Size, Frame Type, and Clamping Pressure on the Ballistic Performance of Soft Body Armor, Composites B, 2008, 39, p 476-489

15. S. Kawabata, M. Niwa, and H. Kawai, The Finite-deformation Theory of Plain-weave Fabrics Part I: The Biaxial-deformation Theory, J. Text. Inst., 1973, 64, p 21-46

16. S. Kawabata, M. Niwa, and H. Kawai, The Finite-deformation Theory of Plain-Weave Fabrics Part II: The Uniaxial-deformation Theory, $J$. Text. Inst., 1973, 64, p 47-61
17. S. Kawabata, M. Niwa, and H. Kawai, The Finite-deformation Theory of Plain-weave Fabrics Part I: The Shear-deformation Theory, J. Text. Inst., 1973, 64, p 62-85

18. I. Ivanov and A. Tabiei, Loosely Woven Fabric Model with Viscoelastic Crimped Fibres for Ballistic Impact Simulations, Int. J. Num. Methods Eng., 2004, 61, p 1565-1583

19. M.J. King, P. Jearanaisilawong, and S. Socrate, A Continuum Constitutive Model for the Mechanical Behavior of Woven Fabrics, Int. J. Solids Struct., 2005, 42, p 3867-3896

20. P. Boisse, B. Zouari, and A. Gasser, A Mesoscopic Approach for the Simulation of Woven Fibre Composite Forming, Comp. Sci. Technol., 2005, 65, p 429-436

21. X. Peng and J. Cao, A Dual Homogenization and Finite Element Approach for Material Characterization of Textile Composites, Compos. B Eng., 2002, 33, p 45-56

22. A. Shahkarami and R. Vaziri, A Continuum Shell Finite Element Model for Impact Simulation of Woven Fabrics, Int. J. Impact Eng., 2007, 34, p 104-119

23. B.R. Scott and C.F. Yen, Analytic Design Trends in Fabric Armor, Proceedings of the 22nd International Ballistics Symposium, 2005, p 752-760

24. ABAQUS version 6.7, User Documentation, Dassault Systems, Providence, RI, 2007

25. D. Starratt, T. Sanders, E. Cepus, A. Poursartip, and R. Vaziri, An Efficient Method for Continuous Measurement of Projectile Motion in Ballistic Impact Experiments, Int. J. Impact Eng., 2000, 24, p 155-170

26. ABAQUS Version 6.7.4, Example Problems Manual, 3.3.1 Seat Belt Analysis of a Simplified Crash Dummy, Dassault Systems, Providence, RI, 2007

27. M. Grujicic, B. Pandurangan, U. Zecevic, K.L. Koudela, and B.A. Cheeseman, Ballistic Performance of Alumina/S-2 Glass-Reinforced Polymer-Matrix Composite Hybrid Lightweight Armor Against Projectiles, Multidiscip. Model Mater. Struct., 2007, 3, p 287-312

28. M. Grujicic, V. Sellappan, G. Arakere, J.C. Ziegert, F.Y. Koçer, and D. Schmueser, Multi-Disciplinary Design Optimization of a Composite Car Door for Structural Performance, NVH, Crashworthiness, Durability and Manufacturability, Multidiscipline Modeling in Materials and Structures, accepted for publication, December 2007 